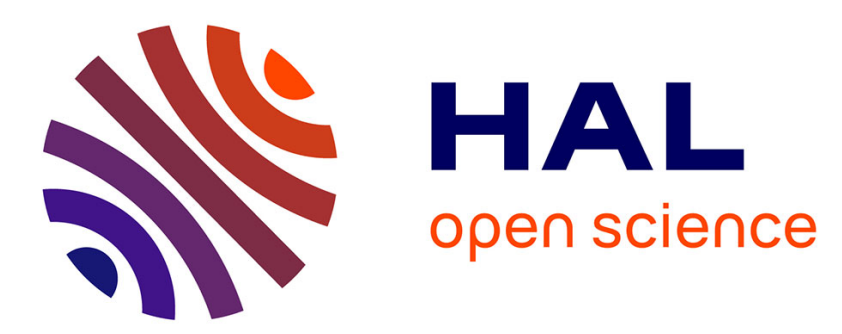

\title{
Model reduction and frequency residuals for a robust estimation of nonlinearities in subspace identification
}

\author{
G. de Filippis, J.P. Noel, G. Kerschen, L. Soria, C. Stephan
}

\section{To cite this version:}

G. de Filippis, J.P. Noel, G. Kerschen, L. Soria, C. Stephan. Model reduction and frequency residuals for a robust estimation of nonlinearities in subspace identification. Mechanical Systems and Signal Processing, 2017, 93, p. 312-331. 10.1016/j.ymssp.2017.01.020 . hal-01570611

\section{HAL Id: hal-01570611 \\ https://hal.science/hal-01570611}

Submitted on 29 Nov 2019

HAL is a multi-disciplinary open access archive for the deposit and dissemination of scientific research documents, whether they are published or not. The documents may come from teaching and research institutions in France or abroad, or from public or private research centers.
L'archive ouverte pluridisciplinaire HAL, est destinée au dépôt et à la diffusion de documents scientifiques de niveau recherche, publiés ou non, émanant des établissements d'enseignement et de recherche français ou étrangers, des laboratoires publics ou privés. 


\title{
Model reduction and frequency residuals for a robust estimation of nonlinearities in subspace identification
}

\author{
G. De Filippis ${ }^{\mathrm{a}, *}$, J.P. Noël ${ }^{\mathrm{b}}$, G. Kerschen ${ }^{\mathrm{b}}$, L. Soria ${ }^{\mathrm{a}}$, C. Stephan ${ }^{\mathrm{c}}$

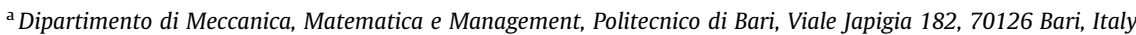 \\ ${ }^{\mathrm{b}}$ Space Structures and Systems Laboratory (S3L), Aerospace and Mechanical Engineering Department, University of Liège, Liège, Belgium \\ c ONERA - The French Aerospace Lab, France
}

\begin{abstract}
The introduction of the frequency-domain nonlinear subspace identification (FNSI) method in 2013 constitutes one in a series of recent attempts toward developing a realistic, firstgeneration framework applicable to complex structures. If this method showed promising capabilities when applied to academic structures, it is still confronted with a number of limitations which needs to be addressed. In particular, the removal of nonphysical poles in the identified nonlinear models is a distinct challenge. In the present paper, it is proposed as a first contribution to operate directly on the identified state-space matrices to carry out spurious pole removal. A modal-space decomposition of the state and output matrices is examined to discriminate genuine from numerical poles, prior to estimating the extended input and feedthrough matrices. The final state-space model thus contains physical information only and naturally leads to nonlinear coefficients free of spurious variations. Besides spurious variations due to nonphysical poles, vibration modes lying outside the frequency band of interest may also produce drifts of the nonlinear coefficients. The second contribution of the paper is to include residual terms, accounting for the existence of these modes. The proposed improved FNSI methodology is validated numerically and experimentally using a full-scale structure, the Morane-Saulnier Paris aircraft.
\end{abstract}

\section{Introduction}

The need to embrace nonlinear behaviour mounts further in industry as increasing technological, economic and environmental pressures are faced. A large body of literature exists regarding the identification and dynamic testing of nonlinear structures, but very little work addresses real-life applications [1]. Most existing methods rely on linearisation or on the definition of equivalent linear modal parameters that vary with the forcing amplitude, as, e.g., described in Ref. [2]. Methods formulated in modal space were also proposed at DLR [3] and by Wright and co-workers [4]. All these approaches have their own merits, specifically they may be compatible with standard test practices. However, they generally assume that the structure under test vibrates in a weakly nonlinear regime of motion, which is a restrictive hypothesis. To offer an example, Weiland and Link proposed a method for the identification of weak nonlinear systems [5,6], based on a linearized set of system's equations, basically derived from the classic Harmonic Balance approach, with assuming that nonlinear terms depend

\footnotetext{
* Corresponding author.

E-mail addresses: giovanni.defilippis@poliba.it (G. De Filippis),jp.noel@ulg.ac.be (J.P. Noël), g.kerschen@ulg.ac.be (G. Kerschen), leonardo.soria@poliba.it (L. Soria), cyrille.stephan@onera.fr (C. Stephan).
} 
individually on each degree-of-freedom response amplitude and that existing coupling effects are negligible. For these reasons, nonlinear system identification has not reached yet the same level of maturity as linear system identification, which is routinely applied to engineering structures [7], in particular to aircraft structures [8].

The introduction of the frequency-domain nonlinear subspace identification (FNSI) method in 2013 [9] constitutes one in a series of recent attempts toward developing a realistic, first-generation framework applicable to complex structures. The FNSI method is a nonlinear generalisation of the well known subspace identification algorithms [10,11], which are widely used for linear system identification [12,13]. The FNSI method derives models of mechanical systems possessing localised nonlinearities directly from measured data and without resorting to a preexisting numerical model, e.g. a finite element model [11]. The method pursues the twofold objective of identifying the underlying linear system, on one side, and, on the other, the lumped nonlinearities. FNSI is applicable to multiple-input, multiple-output structures with high nonproportional damping and high modal density, and makes no assumption as to the importance of nonlinearity in the measured dynamics [9]. The key to the method is the interpretation of nonlinear forces as feedback forces applied to the underlying linear structure, which allows high-dimensional inverse problems and strongly nonlinear regimes of motion to be tackled. The effects of lumped nonlinear inputs in the system are represented by using a linear-in-the-parameters model of the essentially nonlinear, i.e. nonlinearizable, restoring force vector encompassing elastic and dissipative contributions, which is, thus, expressed by a linear combination of suitable nonlinear basis functions with associated nonlinear coefficients. FNSI was validated numerically using simple [9] and more realistic systems [14], and experimentally using an academic setup [15].

There is still a number of limitations in the FNSI method which needs to be addressed. In particular, the removal of nonphysical poles in the identified nonlinear models is a distinct challenge. Similarly to linear system identification, the order of the model is selected in the FNSI approach using a so-called stabilisation diagram. As the model order is increased, the diagram features nonphysical poles, mainly resulting from linear modelling errors, i.e. an overestimation of the model order, nonlinear modelling errors, and noise perturbations. These poles have been shown to strongly perturb the estimation of the nonlinear coefficients [16]. A recent and effective procedure to deal with spurious poles in nonlinear subspace identification is due to Marchesiello and co-workers [17]. They interpret nonlinear coefficients as the ratio of two so-called extended frequency response functions, and perform truncated modal expansions of its numerator and denominator. This allows nonphysical modes to be eliminated during the computation of the nonlinear coefficients. The drawback of this procedure is that spurious poles are not eliminated from the original state-space model and, hence, contaminate model-based output simulations.

In the present paper, to carry out spurious pole removal, we propose, as a first contribution, to operate directly on the identified state-space matrices. A modal-space decomposition of the state and output matrices is examined to discriminate genuine from numerical poles, prior to estimating the extended input and feedthrough matrices. The final state-space model thus contains physical information only and naturally leads to nonlinear coefficients free of spurious variations. Besides spurious variations due to nonphysical poles, vibration modes lying outside the frequency band of interest may also produce drifts of the nonlinear coefficients. The second contribution of the paper is, therefore, to include residual terms accounting for the existence of these modes.

The paper is organised as follows. In Section 2, the removal of spurious poles in modal space and the introduction of lower and upper frequency residuals are discussed in detail. In Section 3, the proposed methodology is numerically validated through the usage of a full-scale structure, the Morane-Saulnier Paris aircraft (see Fig. 1). This aircraft features nonlinear bolted connections, modelled herein as cubic stiffness characteristics. Experimental data acquired on the aircraft structure are finally exploited in Section 4 to demonstrate the methodology in the presence of nonlinear modelling errors and noise. The conclusions of the study are summarised in Section 5.

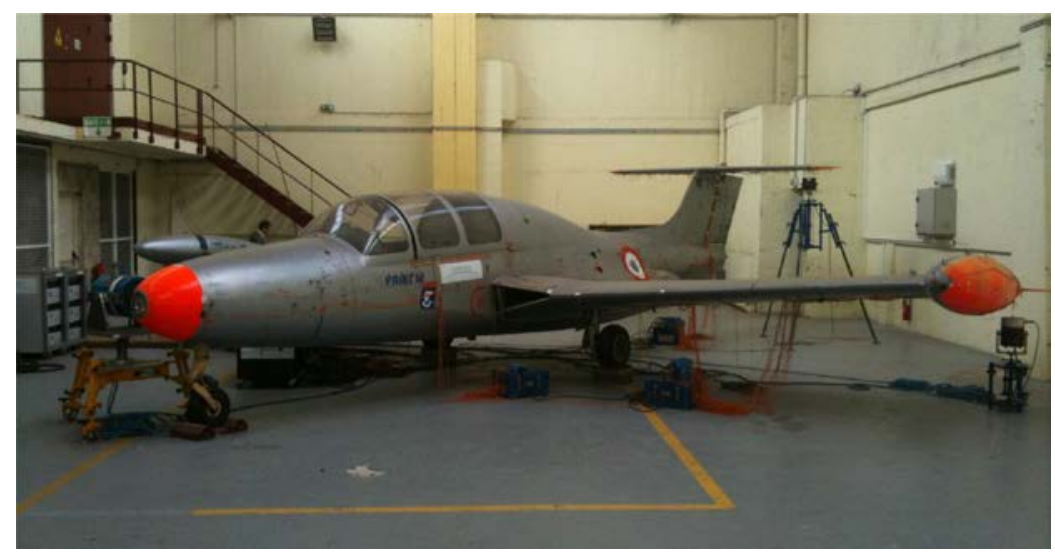

Fig. 1. The Morane-Saulnier Paris aircraft at ONERA's Laboratory. 


\section{An enhanced framework for nonlinear subspace identification}

The mechanical vibration of a dynamic system possessing lumped nonlinearities is governed by

$$
\boldsymbol{M} \ddot{\mathbf{q}}(t)+\boldsymbol{C} \dot{\boldsymbol{q}}(t)+\boldsymbol{K} \boldsymbol{q}(t)+\mathbf{g}(\boldsymbol{q}(t), \dot{\boldsymbol{q}}(t))=\boldsymbol{B}_{u} \boldsymbol{u}(t)
$$

where $\boldsymbol{M}, \boldsymbol{C}, \boldsymbol{K} \in \mathbb{R}^{N \times N}$ are the mass, linear viscous damping and linear stiffness matrices, respectively; $\boldsymbol{q}(t) \in \mathbb{R}^{N \times 1}$ is the generalised displacement vector; $\boldsymbol{B}_{u} \in \mathbb{R}^{N \times N_{i}}$ is a matrix that specifies the location of the $N_{i}$ inputs collected in the vector $\boldsymbol{u}(t) \in \mathbb{R}^{N_{i} \times 1} ; \mathbf{g}(t) \in \mathbb{R}^{N \times 1}$ is the essentially nonlinear, i.e. non-linearisable, restoring force vector encompassing elastic and dissipative contributions; superimposed dots denote differentiations with respect to the time variable $t$; and $N$ is the number of degrees of freedom (DoFs) of the structure after spatial discretisation. The amplitude, direction, location and frequency content of the excitation $\boldsymbol{u}(t)$ determine in which regime, linear or nonlinear, the structure behaves. The effects of the $r$ lumped nonlinear components in the system are represented by using a linear-in-the-parameters model of the form

$$
\boldsymbol{g}(\boldsymbol{q}(t), \dot{\boldsymbol{q}}(t))=\sum_{\alpha=1}^{r} \boldsymbol{b}_{\alpha} \sum_{\beta=1}^{s_{\alpha}} c_{\alpha \beta} h_{\alpha \beta}(\boldsymbol{q}(t), \dot{\boldsymbol{q}}(t))
$$

where $s_{\alpha}$ is the number of nonlinear basis functions $h_{\alpha \beta}(\boldsymbol{q}(t), \dot{\boldsymbol{q}}(t))$ selected to describe the $\alpha$-th nonlinearity, $c_{\alpha \beta}$ are the associated coefficients, and nonlinearity location is specified by the vector $\boldsymbol{b}_{\alpha} \in \mathbb{R}^{N \times 1}$. The total number of nonlinear basis functions introduced in the model is equal to $N_{b}=\sum_{\alpha=1}^{r} s_{\alpha}$. Linearity in the parameters allows for avoiding an iterative optimization process and possible issues related to its initialisation and convergence.

A block-oriented interpretation of nonlinear structural dynamics [18] is obtained by moving the nonlinear internal forces in Eq. (1) to right-hand side and viewing them as feedback forces applied to the underlying linear system, that is

$$
\begin{aligned}
& \boldsymbol{M} \ddot{\boldsymbol{q}}(t)+\boldsymbol{C} \dot{\boldsymbol{q}}(t)+\boldsymbol{K} \boldsymbol{q}(t)=\boldsymbol{B}_{u} \boldsymbol{u}(t)-\sum_{\alpha=1}^{r} \boldsymbol{b}_{\alpha} \sum_{\beta=1}^{s_{\alpha}} c_{\alpha \beta} h_{\alpha \beta}(\boldsymbol{q}(t), \dot{\boldsymbol{q}}(t))=\left[\begin{array}{llll}
\boldsymbol{B}_{u} & c_{11} \boldsymbol{b}_{1} & \cdots & c_{r r_{r}} \boldsymbol{b}_{r}
\end{array}\right] \boldsymbol{e}(t) \\
& \boldsymbol{e}(t)=\left[\begin{array}{llll}
\boldsymbol{u}^{T} & -h_{11} & \cdots & -h_{r s_{r}}
\end{array}\right]^{T}
\end{aligned}
$$

where $\boldsymbol{e}(t) \in \mathbb{R}^{N_{i}+N_{b} \times 1}$ is an extended input vector that concatenates the external forces $\boldsymbol{u}(t)$ and the nonlinear basis functions $h_{\alpha \beta}(t)$.

\subsection{Classical subspace-based methods}

In a standard measurement setup, only $N_{o} \leqslant N$ DoFs are measured. Considering that these outputs $\boldsymbol{y}(t) \in \mathbb{R}^{N_{o} \times 1}$ can be recorded as displacement, velocity or acceleration signals, we obtain

$$
\boldsymbol{y}(t)=\boldsymbol{C}_{d} \boldsymbol{q}(t)+\boldsymbol{C}_{v} \dot{\boldsymbol{q}}(t)+\boldsymbol{C}_{a} \ddot{\boldsymbol{q}}(t)
$$

where $\boldsymbol{C}_{d}, \boldsymbol{C}_{v}$, and $\boldsymbol{C}_{a} \in \mathbb{R}^{N_{0} \times N}$ are the output location matrices for displacement, velocity and acceleration, respectively. Accordingly, the nonlinear basis functions depend on the outputs, i.e. $h_{\alpha \beta}(\boldsymbol{y}(t))$. Defining the state vector $\boldsymbol{x}=\left[\begin{array}{ll}\boldsymbol{q}^{T} & \dot{\boldsymbol{q}}^{T}\end{array}\right]^{T} \in \mathbb{R}^{N_{m} \times 1}$, where the symbol $(\cdot)^{T}$ denotes matrix transposition, Eq. (3) can be recast in the continuous-time state space form

$$
\left\{\begin{array}{l}
\dot{\boldsymbol{x}}(t)=\boldsymbol{A}_{c} \boldsymbol{x}(t)+\boldsymbol{B}_{c}^{e} \boldsymbol{e}(t) \\
\boldsymbol{y}(t)=\boldsymbol{C}_{c} \boldsymbol{x}(t)+\boldsymbol{D}_{c}^{e} \boldsymbol{e}(t)
\end{array}\right.
$$

where the matrices $\boldsymbol{A}_{c} \in \mathbb{R}^{N_{m} \times N_{m}}, \boldsymbol{B}_{c}^{e} \in \mathbb{R}^{N_{m} \times N_{i}+N_{b}}, \boldsymbol{C}_{c} \in \mathbb{R}^{N_{o} \times N_{m}}$, and $\boldsymbol{D}_{c}^{e} \in \mathbb{R}^{N_{o} \times N_{i}+N_{b}}$ are the state, extended input, output and extended direct feedthrough matrices, with dimension of the state space $N_{m}=2 \mathrm{~N}$. The subscript $c$ recalls that a continuous time formulation is considered. State-space and physical-space matrices correspond through the relations

$$
\begin{aligned}
& \boldsymbol{A}_{c}=\left[\begin{array}{cc}
\mathbf{0}^{N \times N} & \boldsymbol{I}^{N \times N} \\
-\boldsymbol{M}^{-1} \boldsymbol{K} & -\boldsymbol{M}^{-1} \boldsymbol{C}
\end{array}\right] \\
& \boldsymbol{B}_{c}^{e}=\boldsymbol{B}_{c}\left[\begin{array}{llll}
\boldsymbol{B}_{u} & c_{11} \boldsymbol{b}_{1} & \cdots & c_{r s_{r}} \boldsymbol{b}_{r}
\end{array}\right] \quad \boldsymbol{B}_{c}=\left[\begin{array}{lll}
\boldsymbol{0}^{N \times N} & \boldsymbol{M}^{-1}
\end{array}\right]^{T} \\
& \boldsymbol{C}_{c}=\left[\begin{array}{llll}
\boldsymbol{C}_{d}-\boldsymbol{C}_{a} \boldsymbol{M}^{-1} \boldsymbol{K} & \boldsymbol{C}_{v}-\boldsymbol{C}_{a} \boldsymbol{M}^{-1} \boldsymbol{C}
\end{array}\right] \\
& \boldsymbol{D}_{c}^{e}=\boldsymbol{D}_{c}\left[\begin{array}{llll}
\boldsymbol{B}_{u} & c_{11} \boldsymbol{b}_{1} & \cdots & c_{r s_{r}} \boldsymbol{b}_{r}
\end{array}\right] \quad \boldsymbol{D}_{c}=\boldsymbol{C}_{a} \boldsymbol{M}^{-1}
\end{aligned}
$$


where $\boldsymbol{B}_{c} \in \mathbb{R}^{N_{m} \times N}$ and $\boldsymbol{D}_{c} \in \mathbb{R}^{N_{0} \times N}$ are the input and direct feedthrough matrices of the underlying linear system, when excited with non zero inputs at each single DoF. We observe that the matrices appearing in the second of Eq. (6) depend on those in the first one, through the following relations

$$
\begin{aligned}
& \boldsymbol{C}_{c}=\left[\begin{array}{ll}
\boldsymbol{C}_{d} & \boldsymbol{C}_{v}
\end{array}\right]+\left[\begin{array}{ll}
\mathbf{0}^{N_{o} \times N} & \boldsymbol{C}_{a}
\end{array}\right] \boldsymbol{A}_{c} \\
& \boldsymbol{D}_{c}^{e}=\left[\begin{array}{ll}
\mathbf{0}^{N_{o} \times N} & \boldsymbol{C}_{a}
\end{array}\right] \boldsymbol{B}_{c}^{e}=\left(\boldsymbol{C}_{c}-\left[\begin{array}{ll}
\boldsymbol{C}_{d} & \boldsymbol{C}_{v}
\end{array}\right]\right) \boldsymbol{A}_{c}^{-1} \boldsymbol{B}_{c}^{e}
\end{aligned}
$$

By Fourier transforming Eq. (6), we obtain the frequency-domain representation of the state space equations

$$
\left\{\begin{array}{l}
\mathrm{i} \omega \boldsymbol{X}(\omega)=\boldsymbol{A}_{c} \boldsymbol{X}(\omega)+\boldsymbol{B}_{c}^{e} \boldsymbol{E}(\omega) \\
\boldsymbol{Y}(\omega)=\boldsymbol{C}_{c} \boldsymbol{X}(\omega)+\boldsymbol{D}_{c}^{e} \boldsymbol{E}(\omega)
\end{array}\right.
$$

where $\omega$ is the angular frequency, $\mathrm{i}=\sqrt{-1}$, and $\boldsymbol{X}(\omega), \boldsymbol{Y}(\omega)$, and $\boldsymbol{E}(\omega)$ are the Fourier transforms of $\boldsymbol{x}(t), \boldsymbol{y}(t)$, and $\boldsymbol{e}(t)$, respectively.

As it is well known, in classical subspace identification, the underlying linear properties are directly computed from the state and output matrices, $\widehat{\boldsymbol{A}}_{c}$ and $\widehat{\boldsymbol{C}}_{c}$, where the symbol $\widehat{(\cdot)}$ is now used to denote the estimated matrices. The extended input and direct feedthrough matrices $\widehat{\boldsymbol{B}}_{c}^{e}$ and $\widehat{\boldsymbol{D}}_{c}^{e}$ are calculated by defining the extended frequency response function (FRF) matrix $\boldsymbol{H}^{e}\left(\omega_{k}\right)$ associated with the state-space model, as

$$
\boldsymbol{H}^{e}\left(\omega_{k}\right)=\hat{\boldsymbol{C}}_{c}\left(\mathrm{i} \omega_{k} \boldsymbol{I}^{N_{m} \times N_{m}}-\hat{\boldsymbol{A}}_{c}\right)^{-1} \hat{\boldsymbol{B}}_{c}^{e}+\hat{\boldsymbol{D}}_{c}^{e}
$$

and minimizing the weighted difference between the measured and modelled output spectra in a least-squares sense

$$
\hat{\boldsymbol{\theta}}_{1}=\arg \min _{\boldsymbol{\theta}_{1}} \sum_{k=1}^{N_{f}} \boldsymbol{W}^{2}\left(\omega_{k}\right)\left|\boldsymbol{Y}\left(\omega_{k}\right)-\boldsymbol{H}^{e}\left(\omega_{k}\right) \boldsymbol{E}\left(\omega_{k}\right)\right|^{2}
$$

where $\omega_{k}$ is the discretised angular frequency, $N_{f}$ indicates the number of samples, and $\boldsymbol{W}\left(\omega_{k}\right)$ is a real-valued weighting function [19]. The unknowns in Eq. (15) can be, e.g., rearranged as follows

$$
\left.\hat{\boldsymbol{\theta}}_{1}=\operatorname{vec}\left[\begin{array}{c}
\hat{\boldsymbol{B}}_{c}^{e} \\
\hat{\boldsymbol{D}}_{c}^{e}
\end{array}\right]\right) \in \mathbb{R}^{\left(N_{m}+N_{o}\right)\left(N_{i}+N_{b}\right) \times 1}
$$

where the vector operation $\operatorname{vec}(\cdot)$ is used to stack the columns of a matrix on top of each other, into a single vector.

\subsection{Role of modal analysis and model reduction}

Since classical nonlinear subspace methods do not provide the user with the possibility of selecting poles computed at a given model order $N_{m}$, discarding the spurious ones is not permitted. The presence of spurious poles can be attributed to many different causes, e.g. noise on the data, modelling errors and computational issues. In the usual procedure, all the poles, stable and unstable, have to be included. To avoid this drawback, we propose a dedicated model reduction procedure. Specifically, we here show how to take advantage of an appropriate coordinate transformation to tackle the analysis of nonlinear systems.

We consider the eigenvalue decomposition of the state matrix $\boldsymbol{A}_{c}$

$$
\boldsymbol{A}_{c}=\boldsymbol{V} \boldsymbol{\Lambda}_{c} \boldsymbol{V}^{-1}
$$

where the eigenvalue matrix $\Lambda_{c} \in \mathbb{C}^{N_{m} \times N_{m}}$ is the well known

$$
\begin{aligned}
& \boldsymbol{\Lambda}_{c}=\left[\begin{array}{cc}
\boldsymbol{\Lambda} & \mathbf{0}^{N \times N} \\
\mathbf{0}^{N \times N} & \boldsymbol{\Lambda}^{*}
\end{array}\right] \\
& \boldsymbol{\Lambda}=\operatorname{diag}\left(\ldots, \lambda_{n}, \ldots\right) \quad \lambda_{n}=-\omega_{n} \zeta_{n}+\mathrm{i} \omega_{n} \sqrt{1-\zeta_{n}^{2}} \text { with } n=1, \ldots, N
\end{aligned}
$$

where $\lambda_{n}$ is the $n$-th pole of the system that contains information about the related natural frequency $\omega_{n}$ and damping ratio $\zeta_{n}$. The symbols $\operatorname{diag}(\cdot)$ and $(\cdot)^{*}$ denote the diagonal matrix and the conjugate operator, respectively. In addition, the eigenvector matrix $\boldsymbol{V} \in \mathbb{C}^{N_{m} \times N_{m}}$ can be written as

$$
\boldsymbol{V}=\left[\begin{array}{cc}
\boldsymbol{\Theta} & \boldsymbol{\Theta}^{*} \\
\boldsymbol{\Theta} \boldsymbol{\Lambda} & \boldsymbol{\Theta}^{*} \boldsymbol{\Lambda}^{*}
\end{array}\right]
$$


where $\Theta \in \mathbb{C}^{N \times N}$ is a matrix whose columns are the modal vectors of the structure under study. Although we are dealing with nonlinear systems, the matrix $\boldsymbol{V}$ can still be utilised to project the equations of motion from the physical to the modal space. The relation between the physical state vector $\boldsymbol{X}(\omega)$ and the modal state vector $\boldsymbol{X}_{m}(\omega)$ is

$$
\boldsymbol{X}(\omega)=\boldsymbol{V} \boldsymbol{X}_{m}(\omega)
$$

Thus, Eq. (13) can be rephrased as

$$
\left\{\begin{array}{l}
\mathrm{i} \omega \boldsymbol{X}_{m}(\omega)=\boldsymbol{\Lambda}_{c} \boldsymbol{X}_{m}(\omega)+\boldsymbol{V}^{-1} B_{c}^{e} \boldsymbol{E}(\omega) \\
\boldsymbol{Y}(\omega)=\boldsymbol{C}_{c} \boldsymbol{V} \boldsymbol{X}_{m}(\omega)+\boldsymbol{D}_{c}^{e} \boldsymbol{E}(\omega)
\end{array}\right.
$$

Accounting for the expression Eq. (11), we can define the modal output matrix $\boldsymbol{\Psi} \in \mathbb{C}^{N_{0} \times N_{m}}$ as

$$
\boldsymbol{\Psi}=\boldsymbol{C}_{c} \boldsymbol{V}=\boldsymbol{\Psi}_{d}+\boldsymbol{\Psi}_{v}+\boldsymbol{\Psi}_{a}
$$

where $\boldsymbol{\Psi}_{d}=\boldsymbol{C}_{d}\left[\begin{array}{ll}\boldsymbol{\Theta} & \boldsymbol{\Theta}^{*}\end{array}\right], \boldsymbol{\Psi}_{v}=\boldsymbol{C}_{v}\left[\begin{array}{ll}\boldsymbol{\Theta} & \boldsymbol{\Theta}^{*} \boldsymbol{\Lambda}^{*}\end{array}\right]$, and $\boldsymbol{\Psi}_{a}=\boldsymbol{C}_{a}\left[\begin{array}{ll}\boldsymbol{\Theta} \boldsymbol{\Lambda}^{2} & \boldsymbol{\Theta}^{*} \boldsymbol{\Lambda}^{* 2}\end{array}\right]$ contain the modal components corresponding to displacement, velocity, and acceleration sensor locations. Similarly, by using the procedure described in Ref. [20], under the assumption of general viscous damping, we find

$$
\boldsymbol{\Phi}^{T}=\boldsymbol{V}^{-1} \boldsymbol{B}_{c}=\boldsymbol{M}_{A}^{-1}\left[\begin{array}{ll}
\boldsymbol{\Theta} & \Theta^{*}
\end{array}\right]^{T}
$$

where $\boldsymbol{M}_{A} \in \mathbb{C}^{N_{m} \times N_{m}}$ is the diagonal matrix commonly referred to as modal A matrix, while the matrix $\boldsymbol{\Phi} \in \mathbb{C}^{N \times N_{m}}$ is the so called modal participation matrix, whose columns are proportional to the modal vectors through the coefficients of $\boldsymbol{M}_{A}$. We observe that, by recalling the definition of $\boldsymbol{B}_{c}^{e}$, given in Eq. (8), this result can be extended to the case of nonlinear systems

$$
\boldsymbol{\Phi}^{e T}=\boldsymbol{V}^{-1} \boldsymbol{B}_{c}^{e}=\boldsymbol{\Phi}^{T}\left[\begin{array}{lllllllll}
\boldsymbol{B}_{u} & c_{11} \boldsymbol{b}_{1} & \cdots & c_{1 s_{1}} \boldsymbol{b}_{1} & \cdots & c_{r 1} \boldsymbol{b}_{r} & \cdots & c_{r s_{r}} \boldsymbol{b}_{r}
\end{array}\right]
$$

We stress that $\boldsymbol{B}_{u}$ and $\boldsymbol{b}_{\alpha}$ (with $\alpha=1, \ldots, r$ ) select the modal components at the input locations and at the position of the $\alpha$-th nonlinearity, respectively, the matrix $\boldsymbol{\Phi}^{e} \in \mathbb{C}^{N_{i}+N_{b} \times N_{m}}$ playing the same role as $\boldsymbol{\Phi}$ in linear modal analysis. Since $\boldsymbol{\Phi}^{e}$ encompasses also the effect of nonlinear internal forces, we denote this term as extended modal participation matrix. In conclusion, the decomposition of the extended direct feedthrough matrix is achieved by inserting the eigenvalue decomposition of $\boldsymbol{A}_{c}$ (Eq. (17)) into Eq. (12)

$$
\boldsymbol{D}_{c}^{e}=\boldsymbol{\Psi}_{a} \boldsymbol{\Lambda}_{c}^{-1} \boldsymbol{\Phi}^{e T}
$$

By accounting for the definitions of Eqs. (23), (25), and (26), the modal state-space model equations can be rewritten as

$$
\left\{\begin{array}{l}
\mathrm{i} \omega \boldsymbol{X}_{m}(\omega)=\boldsymbol{\Lambda}_{c} \boldsymbol{X}_{m}(\omega)+\boldsymbol{\Phi}^{e T} \boldsymbol{E}(\omega) \\
\boldsymbol{Y}(\omega)=\boldsymbol{\Psi} \boldsymbol{X}_{m}(\omega)+\boldsymbol{\Psi}_{a} \boldsymbol{\Lambda}_{c}^{-1} \boldsymbol{\Phi}^{e T} \boldsymbol{E}(\omega)
\end{array}\right.
$$

that can be combined to obtain a linear frequency-domain relationship between input and output Fourier spectra

$$
\begin{aligned}
& \boldsymbol{Y}(\omega)=\boldsymbol{H}^{e}(\omega) \boldsymbol{E}(\omega) \\
& \boldsymbol{H}^{e}(\omega)=\left(\boldsymbol{\Psi}\left(\mathrm{i} \omega \boldsymbol{I}^{N_{m} \times N_{m}}-\boldsymbol{\Lambda}_{c}\right)^{-1}+\boldsymbol{\Psi}_{a} \boldsymbol{\Lambda}_{c}^{-1}\right) \boldsymbol{\Phi}^{e T}
\end{aligned}
$$

where the matrix $\boldsymbol{H}^{e}(\omega) \in \mathbb{C}^{N_{o} \times N_{i}+N_{b}}$ extends the concept of FRF to nonlinear mechanical systems.

The modal state-space model comprising Eq. (27) allows for eliminating the contributions of certain poles/modes by model reduction. Specifically, by this procedure, we reduce the dimensionality of the state vector $\boldsymbol{X}_{m}(\omega)$ from $N_{m}$ to $N_{s}=N_{m}-N_{r}$, where the subscripts $s$ and $r$ denote vectors/matrices whose entries/columns correspond to selected and removed poles, respectively. The contributions of the two different mode sets can be highlighted as follows

$$
\left\{\begin{array}{l}
\mathrm{i} \omega\left[\begin{array}{l}
\boldsymbol{X}_{m s}(\omega) \\
\boldsymbol{X}_{m r}(\omega)
\end{array}\right]=\left[\begin{array}{cc}
\boldsymbol{\Lambda}_{c s} & \mathbf{0}^{N_{s} \times N_{r}} \\
\mathbf{0}^{N_{r} \times N_{s}} & \boldsymbol{\Lambda}_{c r}
\end{array}\right]\left[\begin{array}{l}
\boldsymbol{X}_{m s}(\omega) \\
\boldsymbol{X}_{m r}(\omega)
\end{array}\right]+\left[\begin{array}{c}
\boldsymbol{\Phi}_{s}^{e T} \\
\boldsymbol{\Phi}_{r}^{e T}
\end{array}\right] \boldsymbol{E}(\omega) \\
\boldsymbol{Y}(\omega)=\left[\begin{array}{ll}
\boldsymbol{\Psi}_{s} & \boldsymbol{\Psi}_{r}
\end{array}\right]\left[\begin{array}{l}
\boldsymbol{X}_{m s}(\omega) \\
\boldsymbol{X}_{m r}(\omega)
\end{array}\right]+\left[\begin{array}{ll}
\boldsymbol{\Psi}_{a s} & \boldsymbol{\Psi}_{a r}
\end{array}\right]\left[\begin{array}{cc}
\boldsymbol{\Lambda}_{c s}^{-1} & \mathbf{0}^{N_{s} \times N_{r}} \\
\mathbf{0}^{N_{r} \times N_{s}} & \boldsymbol{\Lambda}_{c r}^{-1}
\end{array}\right]\left[\begin{array}{c}
\boldsymbol{\Phi}_{s}^{e T} \\
\boldsymbol{\Phi}_{r}^{e T}
\end{array}\right] \boldsymbol{E}(\omega)
\end{array}\right.
$$

Once $N_{s} \leqslant N_{m}$ poles are selected, the corresponding diagonal terms in $\hat{\boldsymbol{\Lambda}}_{c}$ and columns in $\hat{\boldsymbol{\Psi}}$, formerly computed by Eqs. (17) and (23), have to be extracted

$$
\begin{aligned}
& \hat{\boldsymbol{\Lambda}}_{c s}=\operatorname{diag}\left(\ldots, \lambda_{j}, \ldots, \lambda_{j}^{*}, \ldots\right) \in \mathbb{C}^{N_{s} \times N_{s}} \\
& \hat{\boldsymbol{\Psi}}_{s}=\left[\begin{array}{lllll}
\cdots & \psi_{p} & \cdots & \psi_{p}^{*} & \cdots
\end{array}\right] \in \mathbb{C}^{N_{0} \times N_{s}} \text { with } p=1, \ldots, N_{s} / 2
\end{aligned}
$$

with $\psi_{p} \in \mathbb{C}^{N_{a} \times 1}$ the $p$-th column of the modal output matrix. Similarly, we obtain $\hat{\mathbf{\Psi}}_{a s} \in \mathbb{C}^{N_{0} \times N_{s}}$ from $\hat{\mathbf{\Psi}}_{a}$. 


\subsection{Frequency residuals}

Since in test campaigns, structures are usually excited over a certain limited frequency range, only the included modes show up clearly in the data, while the influence of modes located in the closeness of the excited band, the so-called outof-band modes, cannot be completely neglected. In the case of linear systems, this effect is modelled by using additional terms [21], whose frequency-dependent behaviour is related to the nature of the measured outputs. Thus, we can write, in a more general form

$$
\boldsymbol{G}(\omega)=\boldsymbol{H}(\omega)+\left((\mathrm{i} \omega)^{-2} \boldsymbol{C}_{d}+(\mathrm{i} \omega)^{-1} \boldsymbol{C}_{v}+\boldsymbol{C}_{a}\right) \boldsymbol{R}_{L}+\left(\boldsymbol{C}_{d}+\mathrm{i} \omega \boldsymbol{C}_{v}+(\mathrm{i} \omega)^{2} \boldsymbol{C}_{a}\right) \boldsymbol{R}_{U}
$$

where $\boldsymbol{G}(\omega)$ and $\boldsymbol{H}(\omega) \in \mathbb{C}^{N_{0} \times N}$ are both linear FRF matrices, the latter encompassing only the set of $N$ modes included in the excited band. The matrices $\boldsymbol{R}_{L}$ and $\boldsymbol{R}_{U} \in \mathbb{R}^{N_{0} \times N}$ are generally referred to as lower and upper frequency residuals. This formulation can be extended to the case of nonlinear systems by considering that

$$
\boldsymbol{H}^{e}(\omega)=\boldsymbol{H}(\omega)\left[\begin{array}{llll}
\boldsymbol{B}_{u} & c_{11} \boldsymbol{b}_{1} & \cdots & c_{r s_{r}} \boldsymbol{b}_{r}
\end{array}\right]
$$

We obtain this expression from Eq. (3), by deriving the relation between outputs and extended inputs in the frequency domain. We stress that substituting $\boldsymbol{H}(\omega)$ with $\boldsymbol{G}(\omega)$ in Eq. (34) leads to the following expression

$$
\begin{gathered}
\boldsymbol{G}^{e}(\omega)=\boldsymbol{H}^{e}(\omega)+\left((\mathrm{i} \omega)^{-2} \boldsymbol{C}_{d}+(\mathrm{i} \omega)^{-1} \boldsymbol{C}_{v}+\boldsymbol{C}_{a}\right) \boldsymbol{R}_{L}^{e}+\left(\boldsymbol{c}_{d}+\mathrm{i} \omega \boldsymbol{C}_{v}+(\mathrm{i} \omega)^{2} \boldsymbol{C}_{a}\right) \boldsymbol{R}_{U}^{e} \\
\boldsymbol{R}_{L}^{e}=\boldsymbol{R}_{L}\left[\begin{array}{llllll}
\boldsymbol{B}_{u} & c_{11} \boldsymbol{b}_{1} & \cdots & c_{r s_{r}} \boldsymbol{b}_{r}
\end{array}\right] \quad \boldsymbol{R}_{U}^{e}=\boldsymbol{R}_{U}\left[\begin{array}{lllll}
\boldsymbol{B}_{u} & c_{11} \boldsymbol{b}_{1} & \cdots & c_{r s_{r}} \boldsymbol{b}_{r}
\end{array}\right]
\end{gathered}
$$

where $\boldsymbol{R}_{L}^{e}$ and $\boldsymbol{R}_{U}^{e} \in \mathbb{R}^{N_{0} \times N_{i}+N_{b}}$ are the extended lower and upper frequency residuals.

We are allowed to draw two considerations: (i) The frequency-dependent behaviour of the residuals is not influenced by the presence of nonlinearity and (ii) the extended lower and upper residuals are obtained as a combination of the linear residual components. Finally, by using the definition of $\boldsymbol{G}^{e}(\omega)$ (Eq. (35)) and considering only the selected modal parameters, we take advantage of the modal decomposition introduced with Eq. (29), obtaining

$$
\boldsymbol{G}^{e}\left(\omega_{k}\right)=\left(\hat{\mathbf{\Psi}}_{s}\left(\mathrm{i} \omega_{k} \boldsymbol{I}^{N_{s} \times N_{s}}-\hat{\boldsymbol{\Lambda}}_{c s}\right)^{-1}+\hat{\mathbf{\Psi}}_{a s} \hat{\boldsymbol{\Lambda}}_{c s}^{-1}\right) \boldsymbol{\Phi}_{s}^{e T}+\left(\left(\mathrm{i} \omega_{k}\right)^{-2} \boldsymbol{C}_{d}+\left(\mathrm{i} \omega_{k}\right)^{-1} \boldsymbol{C}_{v}+\boldsymbol{C}_{a}\right) \boldsymbol{R}_{L}^{e}+\left(\boldsymbol{C}_{d}+\mathrm{i} \omega_{k} \boldsymbol{C}_{v}+\left(\mathrm{i} \omega_{k}\right)^{2} \boldsymbol{C}_{a}\right) \boldsymbol{R}_{U}^{e}
$$

The matrices $\hat{\boldsymbol{\Phi}}_{s}^{e} \in \mathbb{C}^{N_{i}+N_{b} \times N_{s}}, \hat{\boldsymbol{R}}_{L}^{e}$, and $\hat{\boldsymbol{R}}_{U}^{e}$ can be then estimated by minimizing the cost function of a linear-in-the parameters least-squares problem, that is

$$
\hat{\boldsymbol{\theta}}_{2}=\arg \min _{\boldsymbol{\theta}_{2}} \sum_{k=1}^{N_{f}} \boldsymbol{W}^{2}\left(\omega_{k}\right)\left|\boldsymbol{Y}\left(\omega_{k}\right)-\boldsymbol{G}^{e}\left(\omega_{k}\right) \boldsymbol{E}\left(\omega_{k}\right)\right|^{2}
$$

where the unknown parameters are, e.g., concatenated as follows

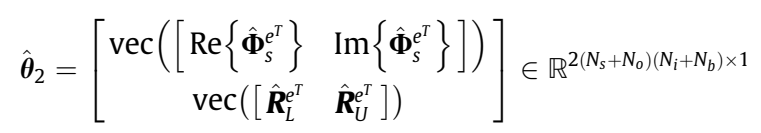

We comment that the least-squares estimator based upon Eq. (38) provides a more general formulation of the estimation problem, with respect to that based on Eq. (15). The analyst is allowed to include the contributions of the selected modal parameters and can decide to account for the effect of lower and upper residuals. Another relevant advantage is that the number of unknowns in $\boldsymbol{\theta}_{2}$ does not depend on the chosen model order $N_{m}$. The resulting computational effort is, thus, only influenced by the number of modes included in the band of analysis.

If no spurious poles appear at the chosen model order, the least-squares problem Eq. (15) remains a good approach to be implemented, in which the matrix $\boldsymbol{D}_{c}^{e}$ can be used for compensating the absence of lower and upper residuals. However, since $\boldsymbol{D}_{c}^{e}$ is a constant matrix, it can only play the role of $\boldsymbol{R}_{L}^{e}$ or of $\boldsymbol{R}_{U}^{e}$, when the measured outputs are all displacements or all accelerations, respectively. In addition, if the effect of the out-of-band modes can be neglected, the unknown parameters in $\boldsymbol{\theta}_{1}$ include only the coefficients of $\boldsymbol{B}_{c}^{e}$. As a consequence, the number of unknowns decreases from $\left(N_{m}+N_{o}\right)\left(N_{i}+N_{b}\right)$ to $N_{m}\left(N_{i}+N_{b}\right)$, with significantly reducing the computational burden of the resulting identification routine.

\subsection{Extraction of nonlinear coefficients}

In the last step of nonlinear subspace methods, the state-space model is converted to physical space, in order to visualize the estimated nonlinear contributions to restoring forces. In Ref. [9] a conversion scheme that relies on Eq. (34) is proposed. With the aim of clarifying its application, we here introduce the example depicted in Fig. 2. This 3 DoF system presents a 


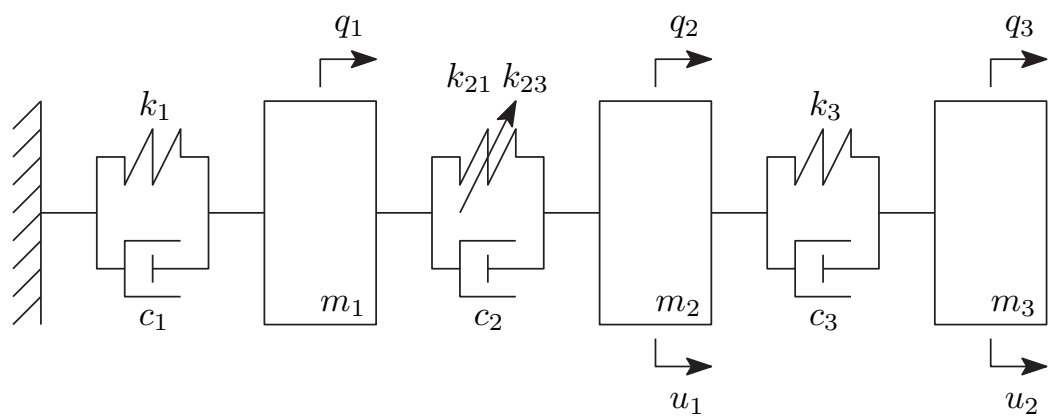

Fig. 2. Example of 3 DoF nonlinear system.

cubic spring between masses $m_{1}$ and $m_{2}$. By using the above introduced notation, the nonlinear restoring force vector can be written as

$$
\mathbf{g}(t)=c_{11} h_{11} \boldsymbol{b}_{1}=k_{23}\left(q_{1}-q_{2}\right)^{3}\left[\begin{array}{lll}
1 & -1 & 0
\end{array}\right]^{T}
$$

the location of the inputs collected in the vector $\boldsymbol{u}(t)=\left[\begin{array}{ll}u_{1} & u_{2}\end{array}\right]^{T}$ specified through the matrix $\boldsymbol{B}_{u}$

$$
\boldsymbol{B}_{u}=\left[\begin{array}{ll}
0 & 0 \\
1 & 0 \\
0 & 1
\end{array}\right]
$$

and, based on the definition given in Eq. (34), the extended FRF matrix $\boldsymbol{H}^{e}(\omega)$ written as

$$
\boldsymbol{H}^{e}(\omega)=\boldsymbol{H}(\omega)\left[\begin{array}{ll}
\boldsymbol{B}_{u} & c_{11} \boldsymbol{b}_{1}
\end{array}\right]=\left[\begin{array}{lll}
H_{12} & H_{13} & k_{23}\left(H_{11}-H_{12}\right) \\
H_{22} & H_{23} & k_{23}\left(H_{12}-H_{22}\right) \\
H_{23} & H_{33} & k_{23}\left(H_{13}-H_{23}\right)
\end{array}\right]
$$

The nonlinear coefficient $k_{23}$ can be extracted from $\boldsymbol{H}^{e}(\omega)$ by using the following formula

$$
k_{23}=\frac{H_{l 3}^{e}}{H_{1 l}-H_{2 l}}
$$

where the index $l=2,3$ depends on the considered input. As a result, the nonlinear coefficients identified from $\boldsymbol{H}^{e}(\omega)$ are spectral quantities, i.e. are complex-valued and frequency-dependent, and can be estimated with respect to different inputs. Moreover, the contribution of each single mode can be taken into account by averaging the obtained values over a frequency band that includes the related natural frequency.

We here present a new conversion scheme that relies on Eq. (25). For the system under study, the extended modal participation matrix $\boldsymbol{\Phi}^{e}$ is given by

$$
\boldsymbol{\Phi}^{e}=\boldsymbol{\Phi}\left[\begin{array}{ll}
\boldsymbol{B}_{u} & c_{11} \boldsymbol{b}_{1}
\end{array}\right]=\left[\begin{array}{ccccc}
\cdots & \phi_{2 p} & \cdots & \phi_{2 p}^{*} & \cdots \\
\cdots & \phi_{3 p} & \cdots & \phi_{3 p}^{*} & \cdots \\
\cdots & k_{23}\left(\phi_{1 p}-\phi_{2 p}\right) & \cdots & k_{23}\left(\phi_{1 p}^{*}-\phi_{2 p}^{*}\right) & \cdots
\end{array}\right]
$$

where the index $p=1,2,3$ indicates the selected mode. By inspecting the entries of $\boldsymbol{\Phi}^{e}$, the nonlinear coefficient $k_{23}$ can be calculated as

$$
k_{23}=\frac{\phi_{3 p}^{e}}{\phi_{1 p}-\phi_{2 p}}
$$

We stress that Eq. (45) cannot be actually used for estimating $k_{23}$, owing to the fact that the term $\phi_{1 p}$ remains not known. This lack of information is frequent in real-life applications and occurs when a structure is not excited at each single DoF. To overcome this drawback, we take advantage of the Maxwell's reciprocity property of linear FRF matrix $\boldsymbol{H}(\omega)$, that implies

$$
H_{j l}=H_{l j} \quad \Longleftrightarrow \quad \psi_{j p} \phi_{l p}=\psi_{l p} \phi_{j p}
$$

where the symbols $\psi_{j p}$ and $\psi_{l p}$ are used to denote the entries of the output modal matrix $\Psi$. Thus, Eq. (45) can be rephrased providing the definitive expression of $k_{23}$

$$
k_{23}=\frac{\phi_{3 p}^{e} \psi_{l p}}{\phi_{l p}\left(\psi_{1 p}-\psi_{2 p}\right)}
$$


As a consequence, following this alternative route, the nonlinear coefficients are identified from $\boldsymbol{\Phi}^{e}$, accounting for the influence of different inputs and modes of vibration.

\section{Numerical demonstration}

According to the theoretical developments of Section 2, an improved FNSI method is proposed in Table 1. If the original FNSI algorithm only accounts for lower or upper frequency residuals through matrix $\boldsymbol{D}_{c}^{e}$, the improved FNSI method removes spurious poles and integrates both lower and upper frequency residuals. The full-scale aircraft structure in Fig. 1 is used in the present section to compare their respective performances. Nonlinearities in the aircraft are located in the front and rear connections between the wings and the fuel tanks, as pictured in Fig. 3.

\subsection{Simulated ground vibration test of the Morane-Saulnier 760 aircraft}

The finite element (FE) model of the MS760 aircraft, shown in Fig. 4, was elaborated from drawings provided by ONERA and is a non-updated version of the real airframe. The wings, T-tail and fuselage were modelled by means of 2-D elements such as beams and shells. Three-dimensional spring elements, which take into account the structural flexibility of the tires and landing gears, were used as boundary conditions. At each wing tip, the front and rear connections between the wings and the fuel tanks were modelled using beam elements. The natural frequencies of the underlying linear system in the $0-55 \mathrm{~Hz}$ frequency range are given in Table 2 . The first six modes correspond to aircraft rigid-body modes, i.e., landing gear suspension modes, whereas the next three modes involve motions of the control surfaces. The first and second wing torsional modes, depicted in Fig. $5 \mathrm{a}$ and b, feature symmetric and antisymmetric wing motions, respectively. These two modes are of particular interest in this study because they involve significant deformation of the nonlinear connections between the wings and fuel tanks. Because the complete FE model possesses more than $8 \times 10^{4}$ DoFs, condensation of the linear

\section{Table 1}

Overview of the proposed subspace identification methodology.

1. Choose the number of processed frequency lines in the measured band and select appropriate basis functions $h_{\alpha \beta}(\boldsymbol{y}(t))$ to represent the nonlinearities

2. Use the state and output matrices, $\boldsymbol{A}_{c}$ and $\boldsymbol{C}_{c}$, resulting from the FNSI algorithm [9] for calculating poles and modal vectors at a given model order

3. Determine the model order $N_{m}$ and select the physical solutions from the stabilisation diagram

4. Apply the reduction scheme based on the modal decomposition (Eq. (30)) and solve the least-squares problem of Eq. (38) to retrieve the extended modal participation matrix $\boldsymbol{\Phi}_{s}^{e}$ and the extended lower and upper residuals, $\boldsymbol{R}_{L}^{e}$ and $\boldsymbol{R}_{U}^{e}$

5. Extract the nonlinear coefficients $c_{\alpha \beta}$ from the extended FRF matrix $\boldsymbol{H}^{e}(\omega)$ or from the extended modal participation matrix $\boldsymbol{\Phi}_{s}^{e}$ (similarly to what done in Eqs. (43) and (47))
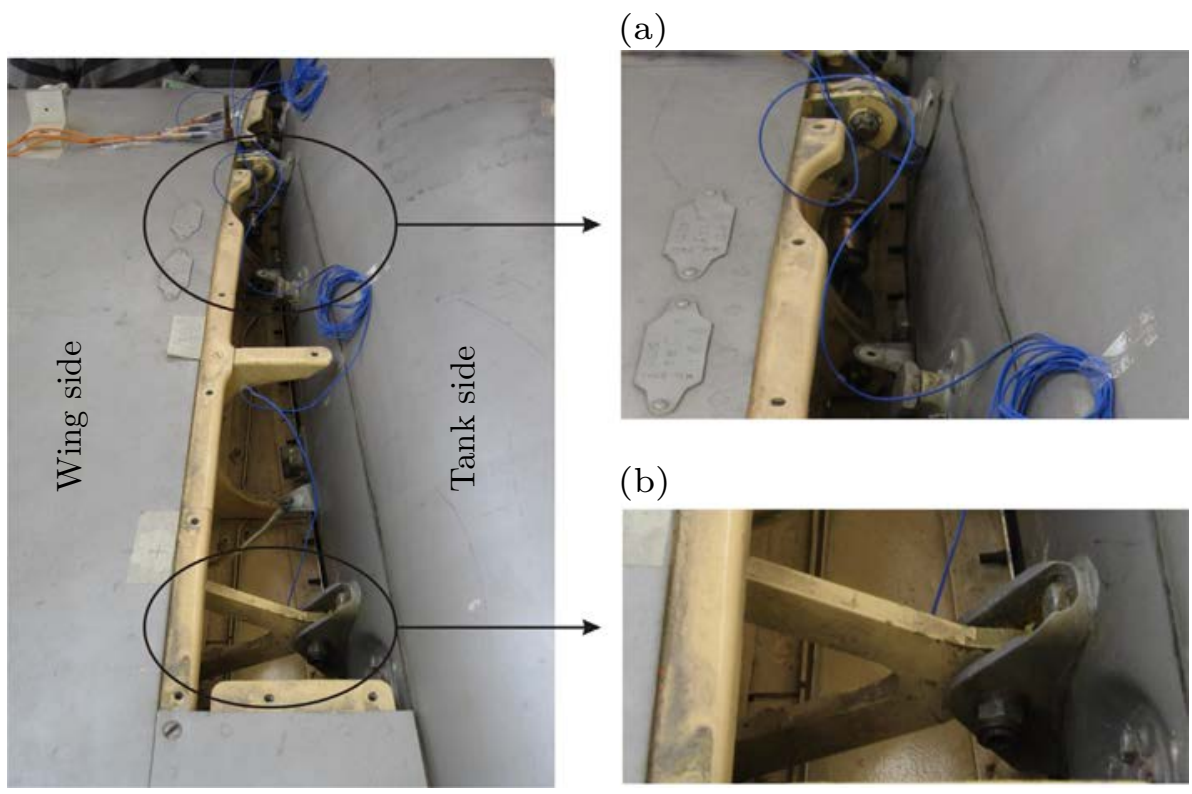

(b)

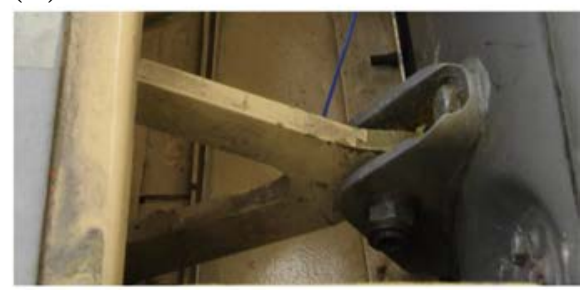

Fig. 3. Connection between external fuel tank and wing tip (top view). Close-up of (a) the front and (b) the rear bolted attachments. 


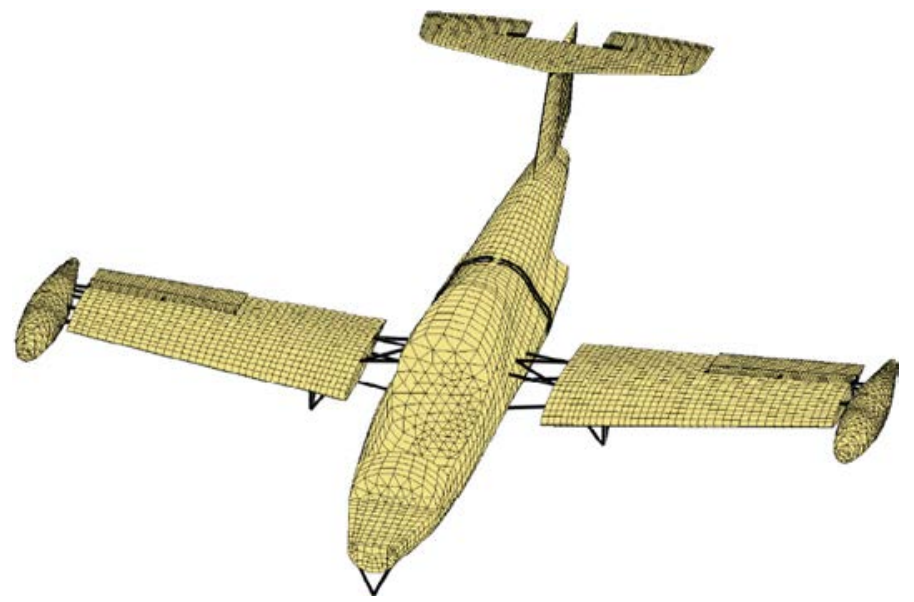

Fig. 4. FE model of the MS760 aircraft.

Table 2

Modal parameters of the reduced-order FE model: the symbol $f_{n}$ denotes the natural frequencies.

\begin{tabular}{|c|c|c|c|c|c|}
\hline Mode & $f_{n}(\mathrm{~Hz})$ & Description & Mode & $f_{n}(\mathrm{~Hz})$ & Description \\
\hline 1 & 0.09 & rigid-body mode & 13 & 21.22 & 1st T-tail sym. bending \\
\hline 2 & 0.73 & rigid-body mode & 14 & 22.77 & front fuselage torsion \\
\hline 3 & 0.96 & rigid-body mode & 15 & 23.65 & T-tail torsion \\
\hline 4 & 1.21 & rigid-body mode & 16 & 25.87 & anti-elevator torsion \\
\hline 5 & 1.22 & rigid-body mode & 17 & 28.27 & 1st fuselage bending \\
\hline 6 & 1.80 & rigid-body mode & 18 & 29.36 & 2nd wing bending \\
\hline 7 & 2.11 & rigid-body mode & 19 & 31.17 & sym. wing torsion \\
\hline 8 & 2.52 & rigid-body mode & 20 & 35.04 & 1st antisym. wing torsion \\
\hline 9 & 3.57 & rigid-body mode & 21 & 39.58 & 3rd wing bending \\
\hline 10 & 8.19 & 1 st wing bending & 22 & 40.87 & 2nd antisym. wing torsion \\
\hline 11 & 9.86 & fin bending & 23 & 47.39 & 2nd fuselage bending \\
\hline 12 & 16.18 & fin torsion & 24 & 52.46 & 2nd T-tail bending \\
\hline
\end{tabular}

(a)

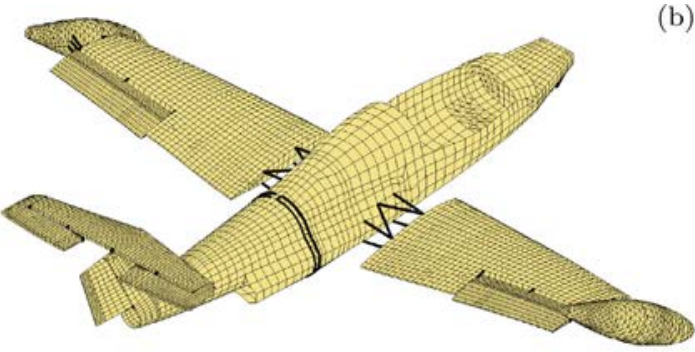

(b)

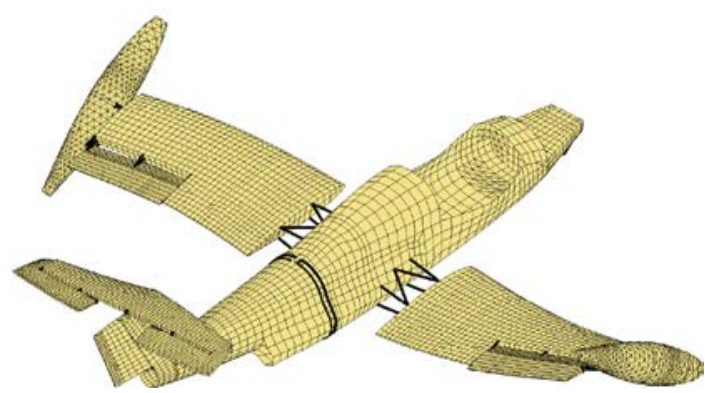

Fig. 5. Mode shapes of the full-order FE model: (a) symmetric wing torsion ( $31.08 \mathrm{~Hz})$ and (b) 1 st antisymmetric wing torsion (34.92 Hz).

components of the model is achieved using the Craig-Bampton reduction technique [22]. Targeting accuracy within the 0$100 \mathrm{~Hz}$ frequency range, 100 internal modes are included in the reduced model. To induce a nonlinear hardening behaviour, two cubic springs with stiffness coefficients of $10^{12} \mathrm{~N} \mathrm{~m}^{-3}$ are introduced at the left and the right front wing-tank connections, along the vertical direction. Proportional damping is added in the FE model, such that the damping ratio of mode 20 is $0.5 \%$. Two excitations in the form of band-limited, normally-distributed random signals are applied to the left and right rear connections along the vertical direction. Their root-mean-square (RMS) amplitude is set to $1000 \mathrm{~N}$ to properly excite the nonlinearities. Numerical simulation is performed using a nonlinear Newmark time integration scheme, and 24 acceleration channels, located at the 8 nodes across the bolted connections, are considered for the identification.

Throughout the paper, the following convention for labelling measurement channels is adopted. The channels related to bolted attachments are denoted by a label of the form $a b c: D$, where $a$ is Ri or Le, for indicating the right or the left side of the aircraft, respectively, $b$ is $F r$ or Re, when referring to the front or the rear bolted connection, respectively, and $c$ is $W i$ or Ta, if the considered node belongs to the wing or to the tank, respectively. Moreover, $D$ represents the channel direction, $X, Y$, or $Z$, 
evaluated with respect to a fixed reference frame. Both ends of the bolted connections are included when letter $c$ is equal to $B C$. For instance, RiFrTa:Z denotes the output channel located at the front of the right wing on the tank side in the vertical direction. For input channels, we only need to distinguish between the left, LeIn:Z, and the right, RiIn:Z, excitations, where In stands for input channel.

\subsection{Identification of the nonlinearities}

To reflect the lack of knowledge inherent to real applications, the correct mathematical form of the nonlinearities, i.e., a cubic law, is not assumed. Instead, splines with 4 equidistant knots on relative displacement are utilised as basis functions to

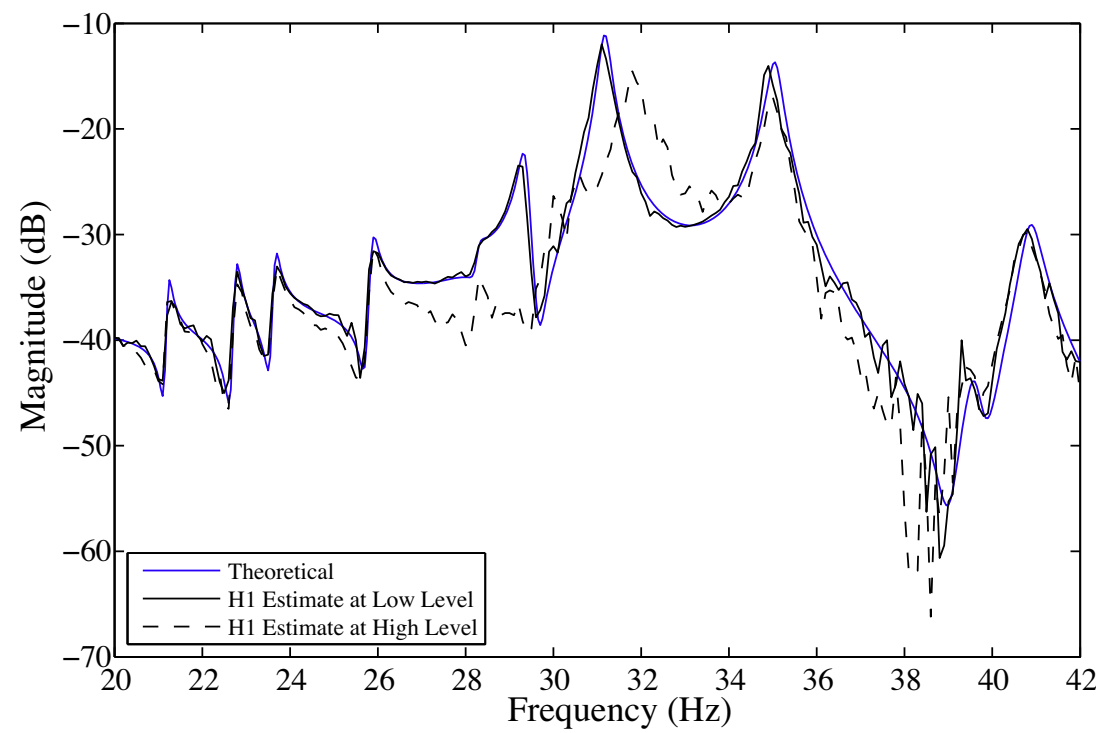

Fig. 6. FE model: Accelerance FRF RiFrTa:Z/RiIn:Z. The theoretical curve of the underlying linear system is given in blue. The solid and dashed curves are obtained through the $\mathrm{H} 1$ estimator. (For interpretation of the references to colour in this figure legend, the reader is referred to the web version of this article.)

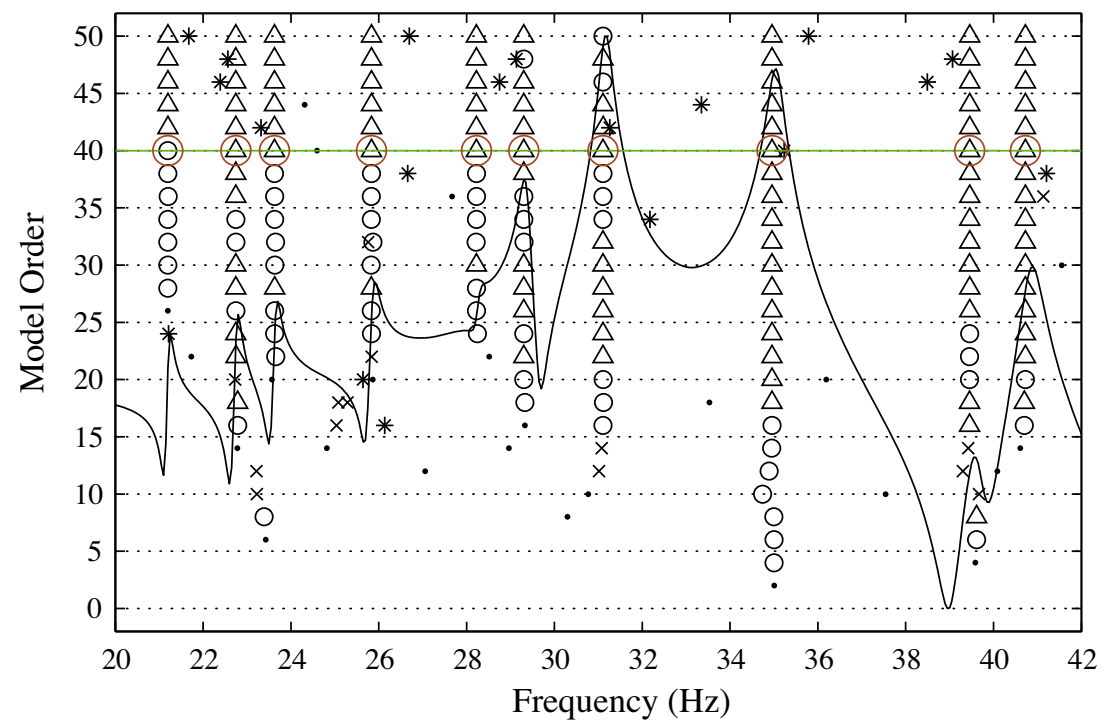

Fig. 7. FE model: Stabilisation diagram computed in the $20-42 \mathrm{~Hz}$ band. Splines with 4 knots on relative displacements of the outputs denoted as RiFrBC:Z

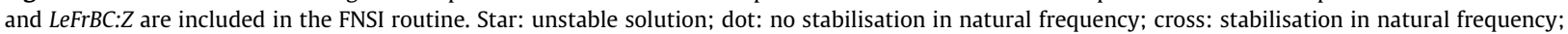
square: extra stabilisation in damping ratio; circle: extra stabilisation in MAC value; triangle: full stabilisation. Stabilisation thresholds for natural frequency, damping ratio and MAC value are $1 \%, 1 \%$ and $99 \%$, respectively. The black curve is the theoretical accelerance FRF RiFrTa:Z/RiIn:Z of the underlying linear system. The green line indicates the chosen model order and the red circles are the selected modal parameters. (For interpretation of the references to colour in this figure legend, the reader is referred to the web version of this article.) 
model the nonlinear restoring forces of the bolted connections, as suggested in [15]. In Fig. 6, to show the system's nonlinear behaviour, we compare the accelerance RiFrTa:Z/RiIn:Z, estimated by using the $\mathrm{H} 1$ algorithm [23], with the theoretical accelerance, computed from the structural matrices. For completeness, the $\mathrm{H} 1$ estimate of the same accelerance in the linear case is even added to Fig. 6; to this aim, the adopted RMS amplitude of low level excitation is set equal to $10 \mathrm{~N}$.

A stabilisation diagram is now used to determine the model order $N_{m}$. This graphical tool assists the analyst in separating the physical system poles from the nonphysical or spurious ones. In practice, the physical poles tend to stabilise when the estimation is performed at increasing model orders. The considered frequency range is $20-42 \mathrm{~Hz}$, because it encompasses the modes which are most affected by the nonlinearities. The stabilisation diagram computed by FNSI is depicted in

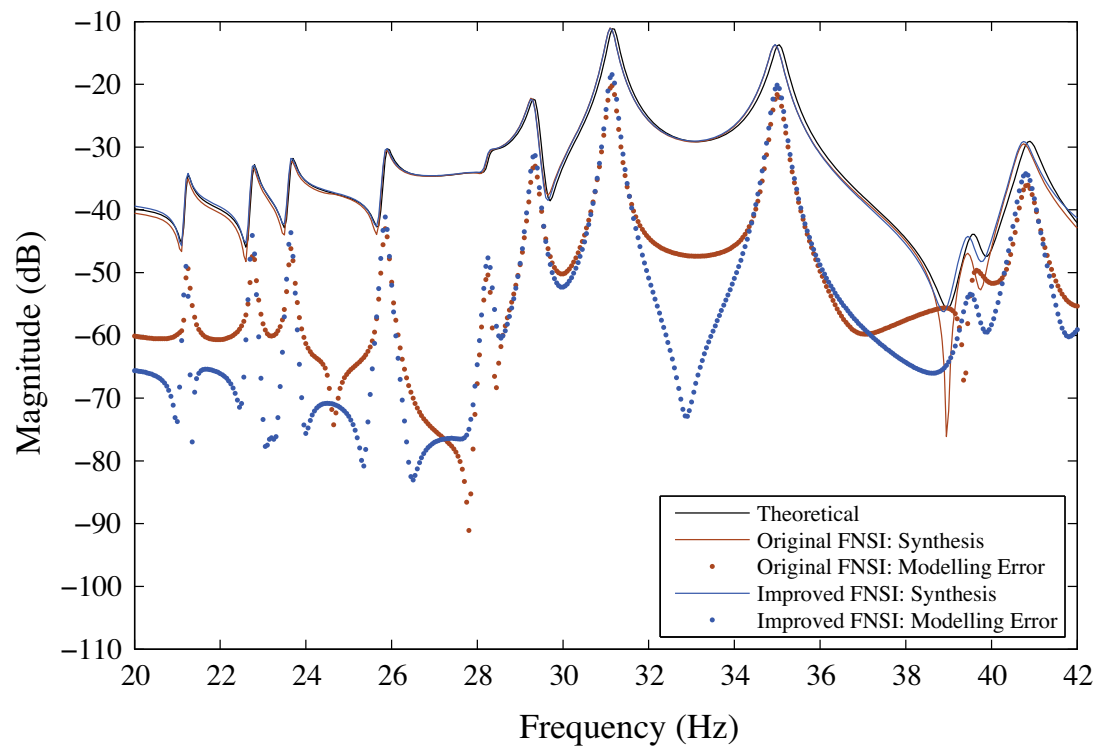

Fig. 8. FE model: Accelerance FRF RiFrTa:Z/RiIn:Z. The theoretical curve of the underlying linear system is given in black. The syntheses resulting from original and improved FNSI are plotted in red and blue, respectively. The modelling error of each synthesis is represented by a dotted line. (For interpretation of the references to colour in this figure legend, the reader is referred to the web version of this article.)
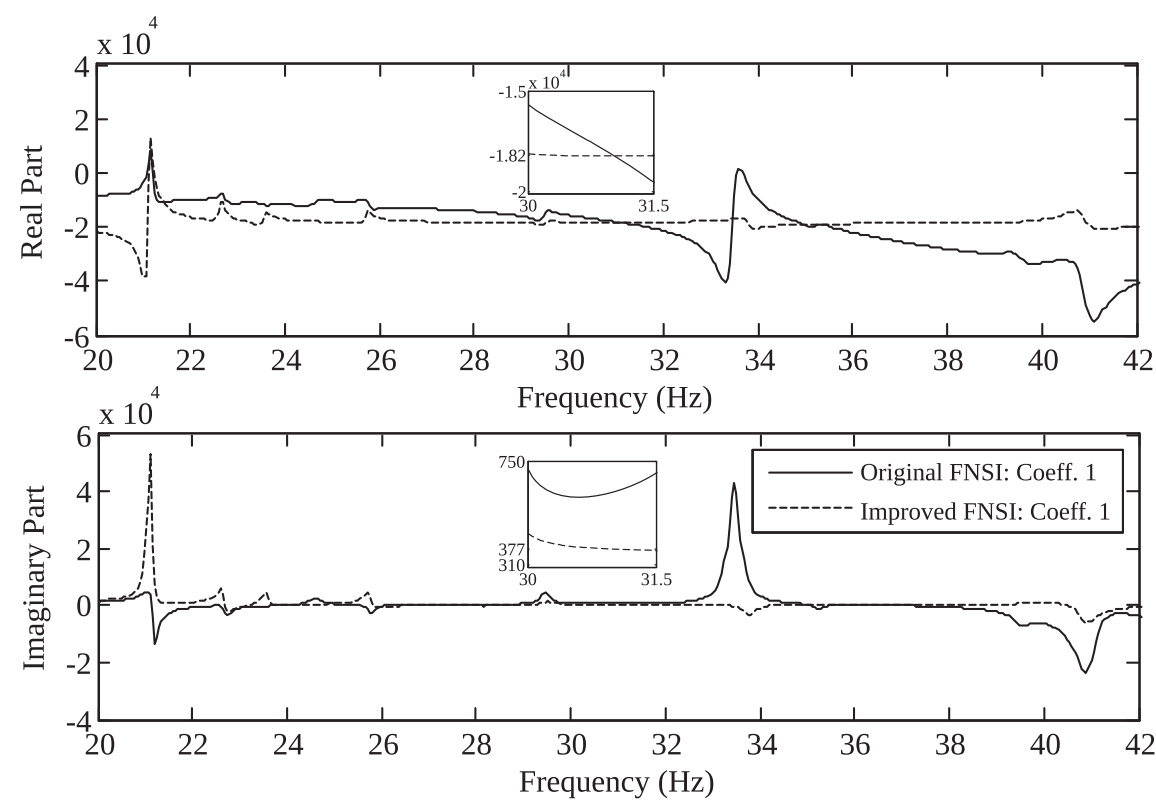

Fig. 9. FE model: Nonlinear coefficient 1 estimated at the RiFrBC:Z/RiIn:Z by using the right input (see following Fig. 10a). The solid and dashed lines refer to the frequency scheme applied to original and improved FNSI methods, respectively. In the insets, an enlarged view of the $30-31.5$ Hz range, encompassing the symmetric wing torsion mode (19 in Table 2), and in which the spectral mean is computed in case of frequency scheme, is offered. The modal scheme value, obtained in combination with the improved FNSI, equal to $-1.82 \times 10^{4}+\mathrm{i} 377$, is indicated on the $y$-axes. 
Fig. 7. Since the simulated data are noiseless, very strict stabilisation tolerances are imposed. It is observed that 10 stabilised solutions (from mode 13 to mode 22 in Table 2, encircled in red in Fig. 7) are computed from order 40, which is then retained in what follows. Specifically, the solution at $21.22 \mathrm{~Hz}$ achieves full stabilisation at this order, while the other 9 stabilise at lower model orders. The presence of two spurious poles, at about $24.5 \mathrm{~Hz}$ and $35.3 \mathrm{~Hz}$ is also noted at this model order.

The accelerances RiFrTa:Z/RiIn:Z of the underlying linear system synthesised using the original FNSI method (Eq. (15)) and its improved version (Eq. (38)) are compared to the theoretical accelerance computed from the structural matrices in Fig. 8. For the improved FNSI method, the two spurious poles are excluded from the analysis. Both synthesised accelerances are in very good agreement with the exact accelerance, which seems to mean that the presence of spurious poles does not significantly affect the FRF estimated from Eq. (15), and the error of the improved FNSI method is bounded almost everywhere by the error of the original method.

(a) RiFrBC:Z (RiIn:Z)

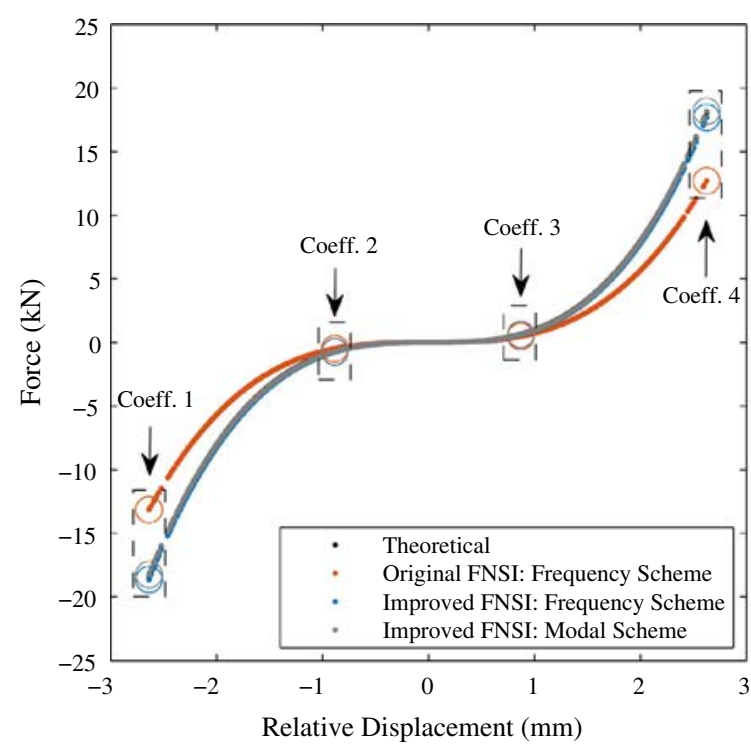

(c) RiFrBC:Z (LeIn:Z)

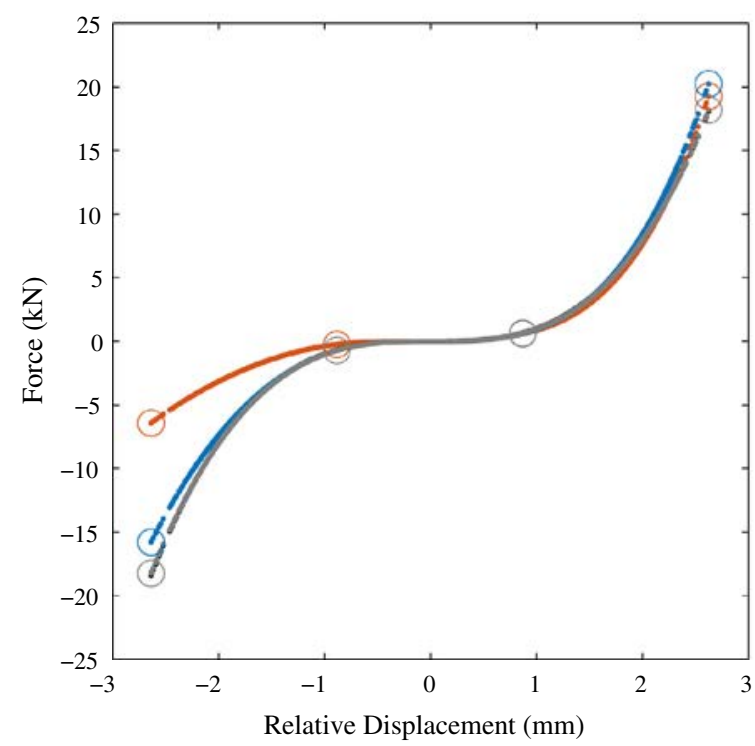

(b) LeFrBC:Z (RiIn:Z)

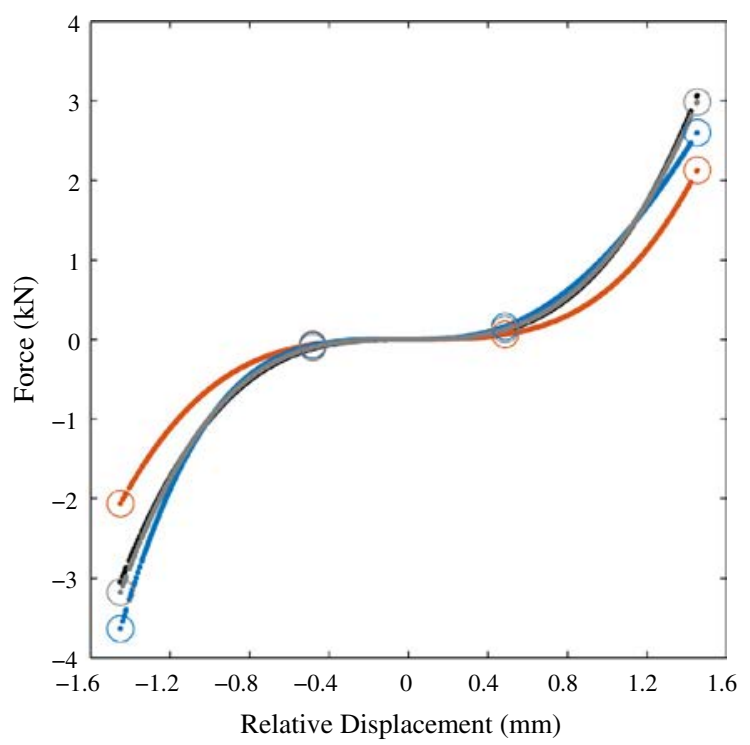

(d) LeFrBC:Z (LeIn:Z)

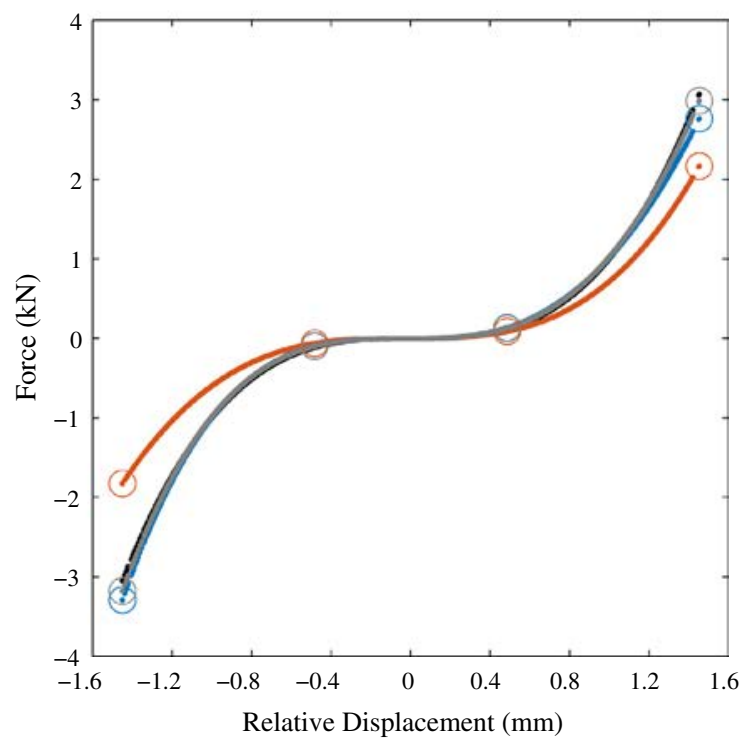

Fig. 10. FE model: Nonlinear stiffness curves. Right reference input (a) and (b); left reference input (c) and (d). The estimate is performed in the $30-31.5 \mathrm{~Hz}$ frequency range, in the closeness of the symmetric wing torsion mode (at $31.17 \mathrm{~Hz}$ in Table 2). The theoretical curves are given in black. The nonlinear restoring forces identified from original FNSI and frequency scheme are depicted in red. The blue and grey curves are obtained by combining the improved FNSI with frequency and modal scheme, respectively. The circles highlight the 4 knots of the splines. (For interpretation of the references to colour in this figure legend, the reader is referred to the web version of this article.) 
The estimation of the nonlinear coefficients with the improved FNSI method can be carried out from the extended FRF matrix (Eq. (43)) or from the extended modal participation matrix (Eq. (47)), which are referred to as frequency or modal scheme, respectively. For the original FNSI method, the estimation is performed using the frequency scheme. For this latter scheme, the nonlinear coefficients are frequency dependent, and a spectral mean has to be calculated. Based on the two schemes, the nonlinear coefficients can be estimated with accounting for the contribution of different inputs and modes of vibration. We expect that improved estimates can be achieved when only the modes that properly activate the nonlinearity are considered. Moreover, we expect an influence related to the inclusion of different inputs depending on their locations. In this study, the 30-31.5 Hz frequency range, which encompasses the symmetric wing torsion mode, is considered. For the modal scheme, only the modal component related to this mode shape is included in the estimation. In Fig. 9, the nonlinear coefficient 1, estimated at location RiFrBC:Z, by using the right input, and resulting from the frequency scheme applied to the original and to the improved FNSI methods, is represented in the entire considered $20-42 \mathrm{~Hz}$ range. We observe that while strong variations of nonlinear coefficient 1 appear where modes not activating the nonlinearity are located, a smooth trend behaviour is noticed for those modes that mostly activate the nonlinearity, which are related to wings deformation. An enlarged view of the chosen $30-31.5 \mathrm{~Hz}$ range, encompassing the symmetric wing torsion mode (19 in Table 2), in which a spectral mean is computed in case of frequency scheme, is even offered in Fig. 9. We find out a lower imaginary part of coefficient 1 , obtained when the improved FNSI is used in combination with the frequency scheme. Moreover, we observe that the computed spectral mean value is close to that of coefficient 1 had by using the modal scheme. Similar considerations hold for all the other nonlinear coefficients, confirming that an enhanced accuracy of the estimation procedure is achieved.

The identified nonlinear stiffness curves, computed with respect to the right and the left input, are compared with the theoretical curve in Fig. 10. Clearly, the improved FNSI method is able to successfully identify the nonlinearities of the bolted connections, with the modal scheme slightly outperforming the frequency scheme. Although no spurious pole is present in the $30-31.5 \mathrm{~Hz}$ frequency range, the improved FNSI method confirms its superiority over the original FNSI method. Specifically, the blue curves, obtained by combining the improved FNSI with the frequency scheme, tend to converge to the right values when the reference inputs are chosen on the same side of nonlinearity locations. Moreover, the grey curves, resulting from the improved FNSI when combined with the modal scheme, end to perfectly overlap the theoretical values. The modal scheme, basically appears less sensitive to the choice of the reference input, thus confirming the robustness of this new approach.

We comment that this improved nonlinear identification is expected to lead to a better reconstruction of output time histories with respect to those computed by the original FNSI procedure, owing to improved time domain analytical models of the systems in study can be had as a final result.

\section{Experimental identification}

Experimental data collected on the MS760 aircraft during a ground vibration testing campaign at ONERA are now exploited. The considered structural configuration is the aircraft without its engines standing on the ground on its three landing gears with deflated tires. The sensor layout comprised a total of 38 accelerometers with 8 tri-axial sensors positioned across the 4 wing-tank bolted attachments (Fig. 11). Two shakers, one visible in Fig. 1, applied band-limited white-noise signals to the two wings in the vertical direction. Low and high forcing levels were considered with a RMS amplitude of $4.4 \mathrm{~N}$ and $43.9 \mathrm{~N}$, respectively. Force signals were recorded by means of impedance heads.

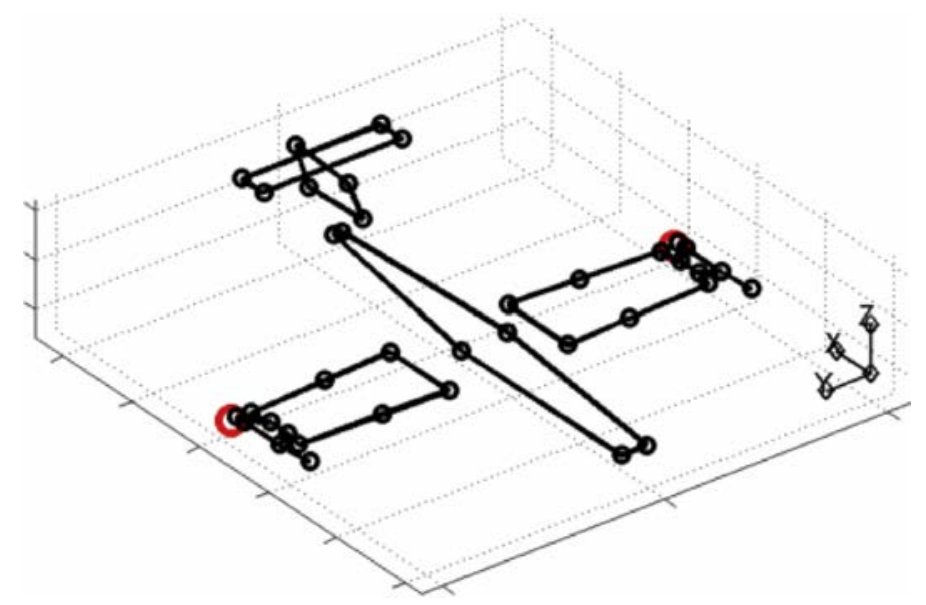

Fig. 11. Layout of the experimental setup. Black circles: output locations; red circles: driving points. (For interpretation of the references to colour in this figure legend, the reader is referred to the web version of this article.) 


\subsection{Linear analysis at low level and nonlinearity detection}

In this section, a classical linear subspace identification is performed on collected data, at low level to estimate the modal parameters of the underlying linear system, and, at high level, for nonlinearity detection purposes. Specifically, the nonlinear restoring force vector $\mathbf{g}(t)$, in Eq. (2), is excluded from the FNSI routine, since we, here, hypothesize that its effect on the dynamics of the system is negligible. At $4.4 \mathrm{~N}$, in fact, the nonlinearities affecting the bolted connections are not properly activated. Fig. 12 shows the stabilisation diagram of the underlying linear aircraft extracted by FNSI in the 7-21.5 Hz frequency range. The five vibration modes displayed in Fig. 15 stabilise and their resonance frequencies and damping ratios are listed in the second and third columns of Table 3. We comment that while the last three solutions stabilise from order 10 , the first two poles are already fully stable at this order. We, morever, stress that the identified modes should not be directly related to those reported in Table 2, being the FE model used in Section 3 a non-updated version of the real airframe. In Fig. 13, a satisfactory agreement between the FRFs synthesised by the original and improved FNSI methods and that obtained with the H1 estimator is observed. We, however, note that the antiresonance around $15.5 \mathrm{~Hz}$ is better captured by the improved FNSI method.

Applying the same linear identification at $43.9 \mathrm{~N}$, the resonance frequencies and damping ratios listed in the fourth and fifth columns of Table 3 are obtained. All resonance frequencies migrate to lower values, unveiling the overall softening behaviour due to loosening in the bolted connections. To quantify the importance of this global effect, percentage discrepancies between values of resonance frequencies and damping ratios attained in the cases of the two levels of excitation are even reported in Table 3. In fact, by comparing modal parameters estimated at low and high level, it is possible to (i) single out those modes responsible for triggering nonlinear mechanisms and (ii) quantify the amount of nonlinearity associated with each mode of vibration. Moreover, the modal assurance criterion (MAC) [23,24] computed between the vibration modes

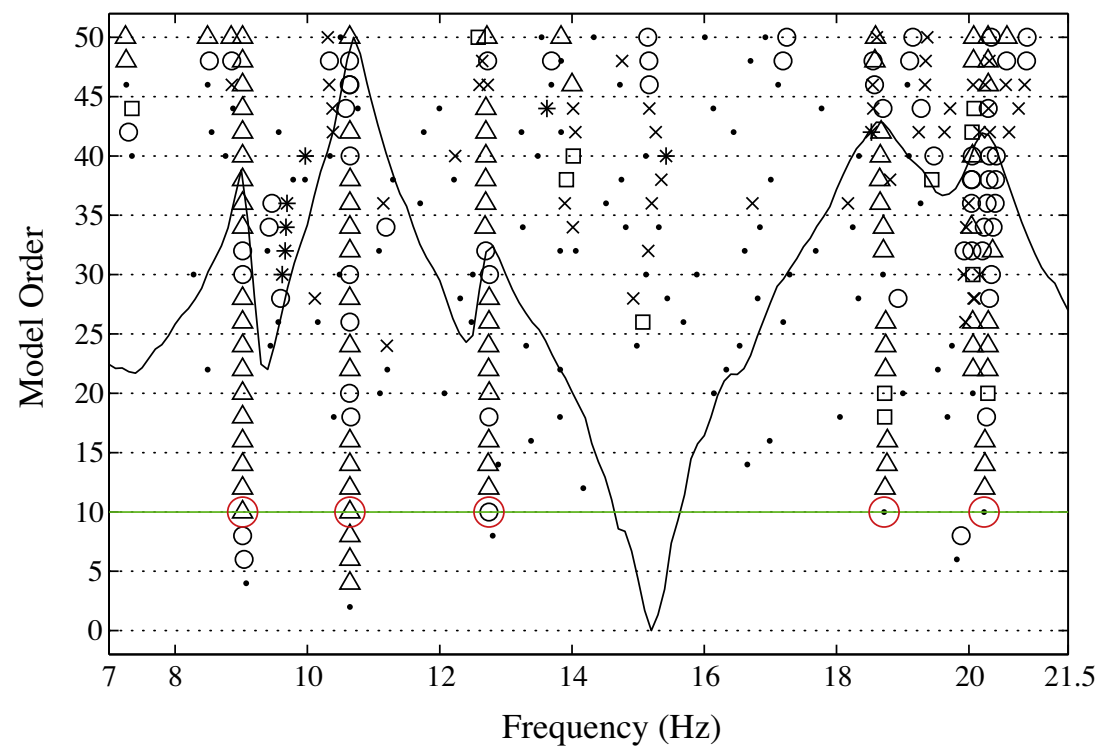

Fig. 12. Experimental model: Stabilisation diagram computed in the $7-21.5 \mathrm{~Hz}$ band. Star, dot, crosses, squares, circles and triangles are defined similarly to Fig. 7. Stabilisation thresholds for natural frequency, damping ratio and MAC value are $1 \%, 5 \%$ and $98 \%$, respectively. The black curve is the accelerance FRF LeReWi:Y/LeIn:Z, obtained with the H1 estimator at low excitation level (4.4 N RMS). The green line indicates the chosen model order and the circles highlight the selected modal parameters. (For interpretation of the references to colour in this figure legend, the reader is referred to the web version of this article.)

Table 3

Comparison between modal parameters estimated at low (4.4 N RMS) and high (43.9 N RMS) excitation levels, through linear subspace identification, performed by running, in both the cases, the FNSI routine with the nonlinear restoring force vector $\mathbf{g}(t)$ suppressed. Estimated natural frequencies $\left(f_{n}\right)$ and damping ratios $\left(\zeta_{n}\right)$ are identified in the $7-21.5 \mathrm{~Hz}$ band.

\begin{tabular}{|c|c|c|c|c|c|c|c|}
\hline \multirow[t]{2}{*}{ Mode } & \multicolumn{2}{|c|}{ Linear Id. at low level } & \multicolumn{2}{|c|}{ Linear Id. at high level } & \multicolumn{2}{|c|}{ Discrepancy } & \multirow[t]{2}{*}{$\operatorname{MAC}(\%)$} \\
\hline & $f_{n}(\mathrm{~Hz})$ & $\zeta_{n}(\%)$ & $f_{n}(\mathrm{~Hz})$ & $\zeta_{n}(\%)$ & $\delta_{f}(\%)$ & $\delta_{\zeta}(\%)$ & \\
\hline 1 & 9.02 & 0.30 & 8.92 & 0.44 & 1.06 & 46.49 & 99.1 \\
\hline 2 & 10.64 & 0.78 & 10.48 & 1.30 & 1.51 & 68.13 & 98.8 \\
\hline 3 & 12.75 & 0.67 & 12.60 & 0.71 & 1.12 & 6.59 & 39.8 \\
\hline 4 & 18.72 & 1.33 & 17.25 & 0.99 & 7.85 & 25.28 & 75.8 \\
\hline 5 & 20.23 & 0.88 & 19.85 & 1.13 & 1.91 & 28.43 & 96.7 \\
\hline
\end{tabular}




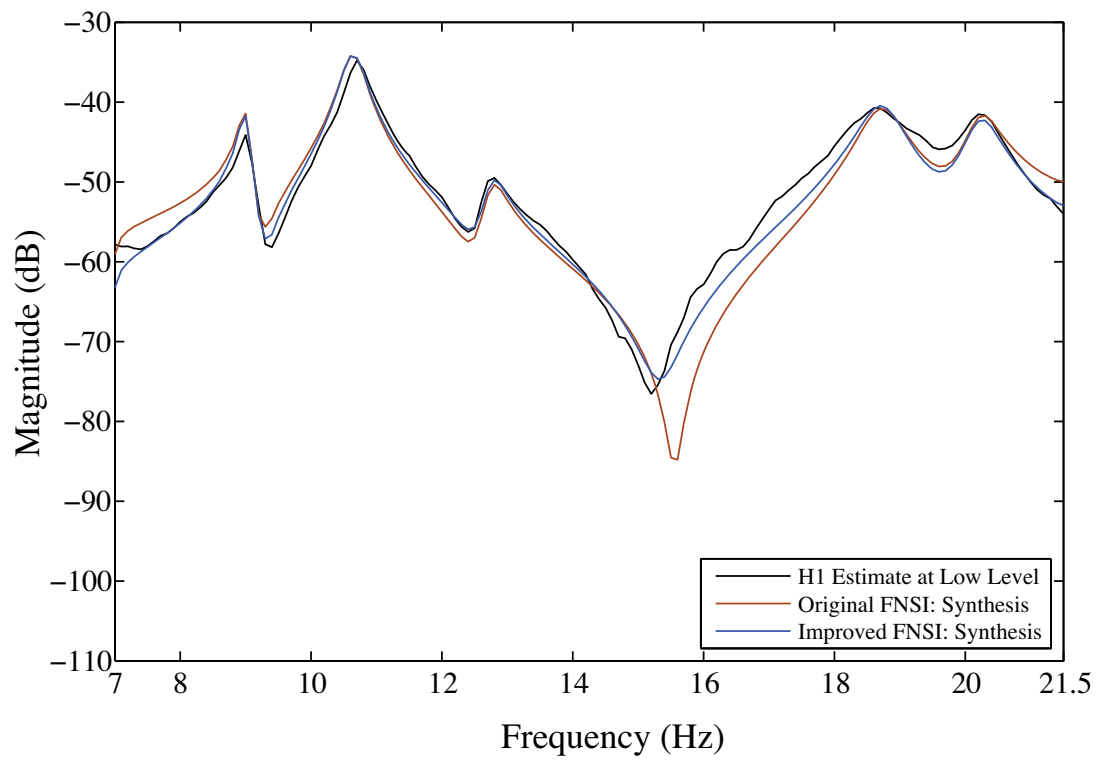

Fig. 13. Experimental model: Accelerance FRF LeReWiY:Z/LeIn:Z computed from low amplitude data (4.4 N RMS). The black curve is obtained with the H1 estimator. Syntheses are defined similarly to Fig. 8. (For interpretation of the references to colour in this figure legend, the reader is referred to the web version of this article.)

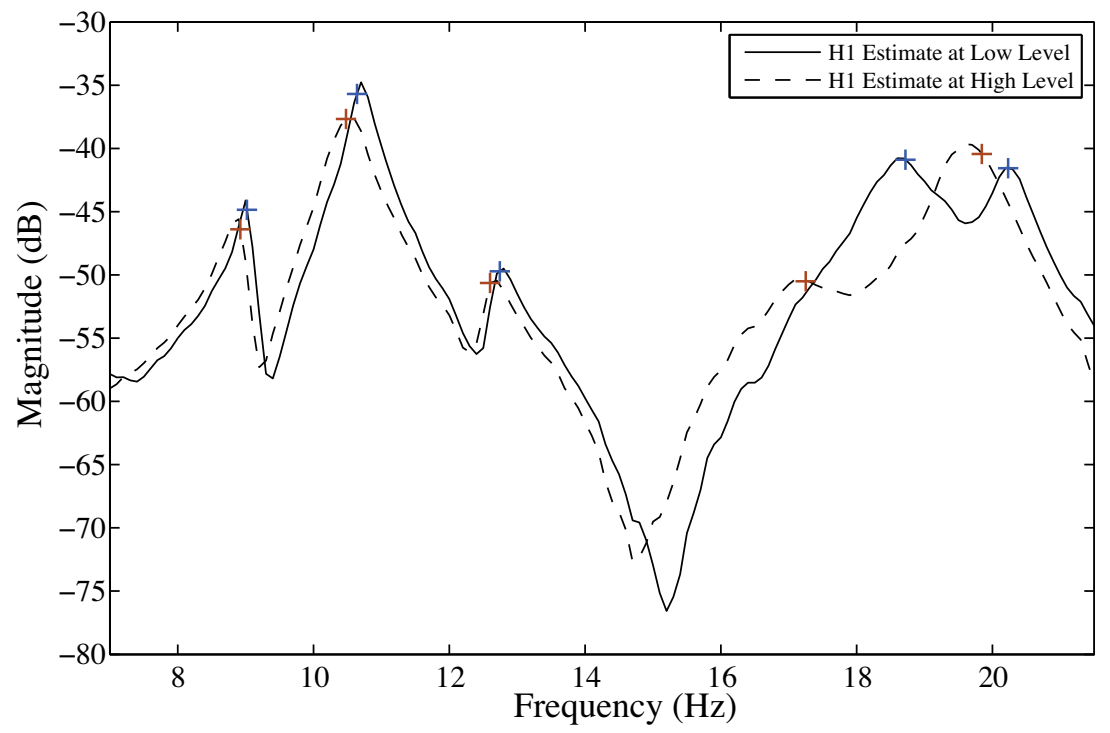

Fig. 14. Experimental model: Accelerance FRF LeReWiY:Z/LeIn:Z. The solid and dashed curves are obtained with the H1 estimator at low (4.4 N RMS) and high (43.9 N RMS) excitation level, respectively. The blue and red markers refer to the natural frequencies listed in the second and fourth columns of Table 3. (For interpretation of the references to colour in this figure legend, the reader is referred to the web version of this article.)

at low and high levels shows that the modal shapes of the third and fourth resonances are strongly affected by the nonlinearities. This significant nonlinear behaviour is confirmed in Fig. 14 which presents the accelerance LeReWi:Y/LeIn:Z calculated with the $\mathrm{H} 1$ estimator at the two excitation levels.

\subsection{Identification at high level}

As for the numerical study, splines are used as basis functions to model the nonlinear behaviour of the four bolted connections. Considering that each bolted connection can potentially have nonlinearities in the three directions, there are 12 candidate nonlinearities, which give rise to a high-dimensional nonlinear identification problem. To facilitate the 
identification process, we have introduced and proposed a new graphical tool [25], that we have named "comparison diagram". Apparently similar to a classic stabilisation diagram, the comparison diagram is obtained by comparing the solutions computed at the different model orders with the underlying linear properties estimated at low excitation level. Thus, by a comparison diagram, comparable model orders and equivalent solutions can be visualised and selected in a very simple and effective way. Specifically, the comparison diagram allows for (i) reducing the dimensionality of the inverse problem by locating the nonlinearities; (ii) evaluating the influence of the selected basis functions on the obtained solutions; and (iii) revealing the presence of the so-called comparable model orders, for which the underlying linear properties are retrieved within certain tolerance limits. Using this approach, we find out that the connections RiReBC:Z, RiFrBC:Z, LeReBC:Y and $L e F r B C: X$ are responsible for activating the dominant nonlinearities, and, therefore, we choose splines with 4 knots on

(a) Mode 1

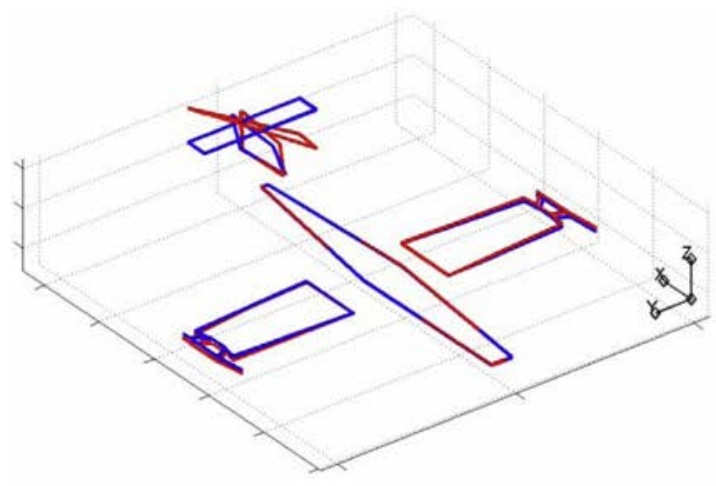

(c) Mode 3

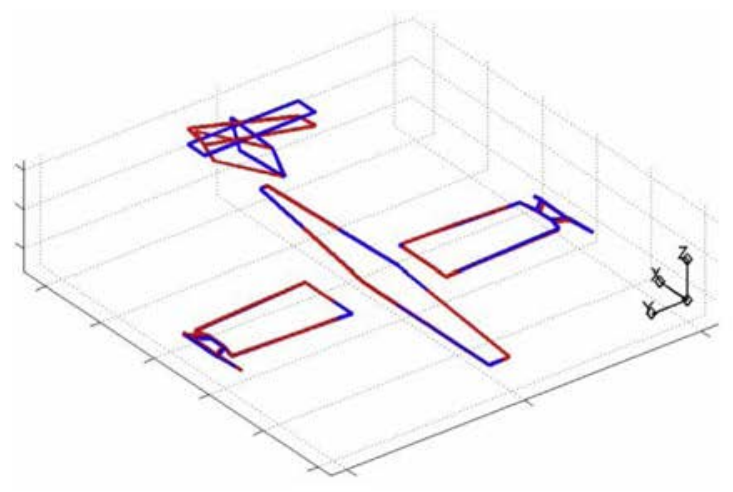

(b) Mode 2

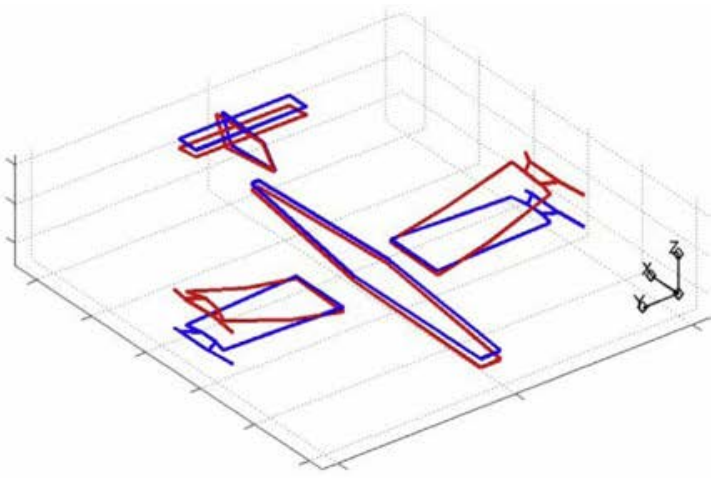

(d) Mode 4

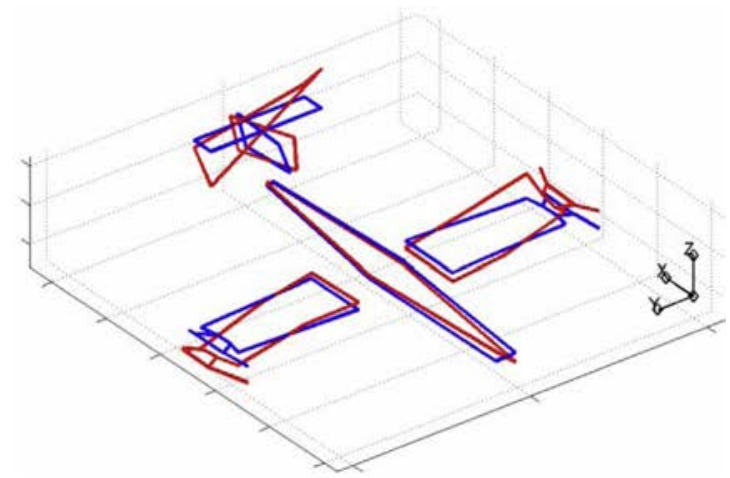

(e) Mode 5

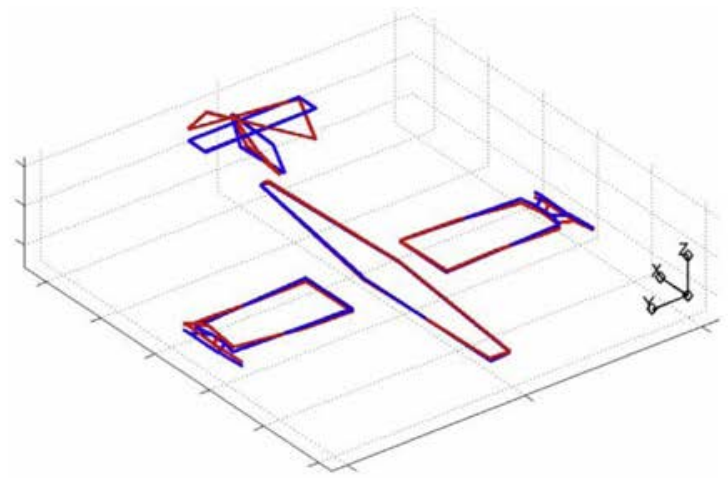

Fig. 15. Experimental model: Mode shapes. Estimates are obtained with the FNSI algorithm at low excitation level (4.4 N RMS). The related natural frequencies and damping ratios are listed in the second and third columns of Table 3. Blue and red lines indicate the undeformed and deformed meshes, respectively. (For interpretation of the references to colour in this figure legend, the reader is referred to the web version of this article.) 


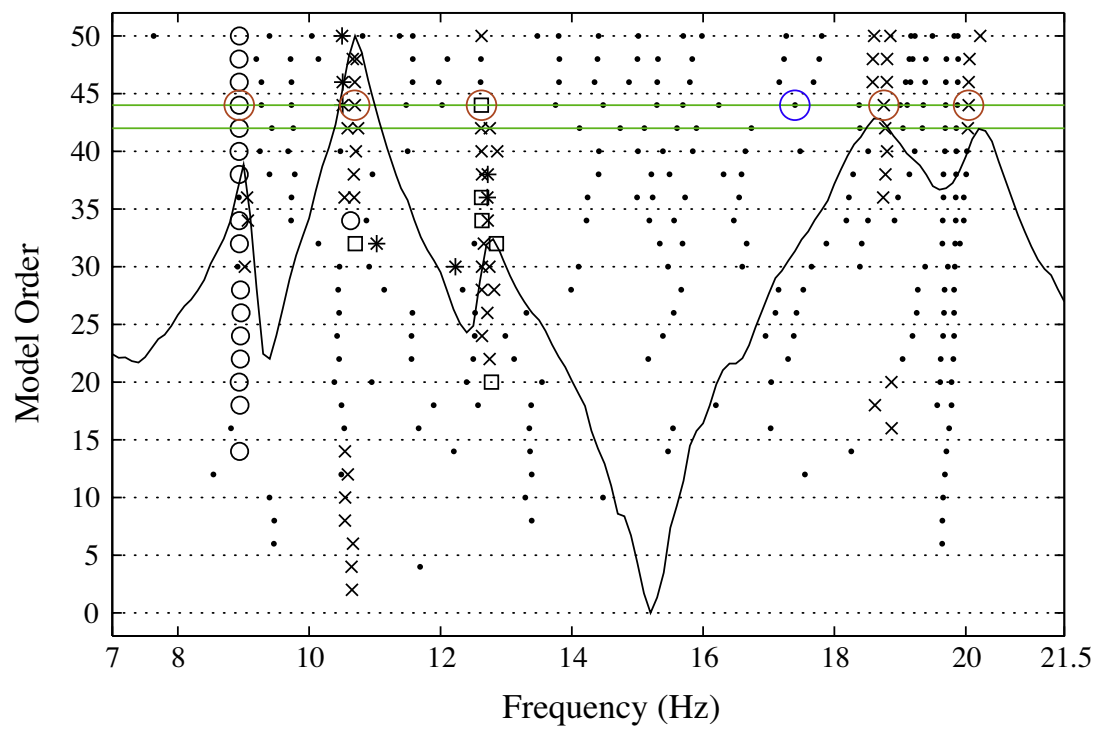

Fig. 16. Experimental model: Comparison diagram computed in the 7-21.5 Hz band. Splines with 4 knots on relative displacements of the outputs denoted as RiReBC:Z, RiFrBC:Z, LeReBC:Y and LeFrBC:X are included in the FNSI routine. Star: unstable solution; dot: no equivalence in natural frequency; cross: equivalence in natural frequency; square: extra equivalence in damping ratio; circle: extra equivalence in MAC value; triangle: full equivalence. Comparison thresholds for natural frequency, damping ratio and MAC value are $1 \%, 5 \%$ and $98 \%$, respectively. The black curve is the accelerance FRF LeReWi: Y/LeIn:Z, obtained with the H1 estimator at low excitation level (4.4 N RMS). The green lines indicate the comparable model orders and the circles highlight the selected modal parameters. (For interpretation of the references to colour in this figure legend, the reader is referred to the web version of this article.)

Table 4

Comparison between modal parameters estimated at low excitation level (4.4 N RMS) by linear subspace identification (see Table 3 ), and at high level (43.9 N RMS) by FNSI. Estimated natural frequencies $\left(f_{n}\right)$ and damping ratios $\left(\zeta_{n}\right)$ are identified in the 7-21.5 $\mathrm{Hz}$ band.

\begin{tabular}{|c|c|c|c|c|c|c|c|}
\hline \multirow[t]{2}{*}{ Mode } & \multicolumn{2}{|c|}{ Linear Id. at low level } & \multicolumn{2}{|c|}{ Nonlinear Id. at high level } & \multicolumn{2}{|l|}{ Error } & \multirow[t]{2}{*}{ MAC (\%) } \\
\hline & $f_{n}(\mathrm{~Hz})$ & $\zeta_{n}(\%)$ & $f_{n}(\mathrm{~Hz})$ & $\zeta_{n}(\%)$ & $\epsilon_{f}(\%)$ & $\epsilon_{\zeta}(\%)$ & \\
\hline 1 & 9.02 & 0.30 & 8.93 & 0.49 & 0.95 & 64.21 & 99.2 \\
\hline 2 & 10.64 & 0.78 & 10.70 & 0.94 & 0.50 & 21.16 & 96.3 \\
\hline 3 & 12.75 & 0.67 & 12.62 & 0.67 & 0.97 & 0.45 & 36.4 \\
\hline \multirow[t]{2}{*}{4} & 18.72 & 1.33 & 17.40 & 1.22 & 7.05 & 8.15 & 87.7 \\
\hline & & & 18.75 & 0.18 & 0.17 & 86.72 & 11.3 \\
\hline 5 & 20.23 & 0.88 & 20.05 & 0.20 & 0.92 & 77.74 & 56.4 \\
\hline
\end{tabular}

relative displacements to model them. The resulting comparison diagram is shown in Fig. 16. Two consecutive model orders, 42 and 44, are found to be comparable, with equivalent solutions encircled in red. An extra equivalence in terms of the MAC value is obtained for the first mode. The underlying linear properties identified by applying FNSI at high level are listed in Table 4. Comparing Tables 3 and 4, a much better estimation of the resonance frequencies of the underlying linear system is observed, with all errors below 1\%. Conversely, the MAC values of modes 4 and 5 are severely degraded, and, overall, the damping ratios are not improved. These errors should be attributed both to the complexity of real bolted connections and to the lack of knowledge about these connections prior to identification. We expect that this behaviour, already pointed out in the detection step (see Section 4.1), could be related to the activation of nonlinear phenomena involving not only the elastic forces, but also the damping ones. Thus, splines are also considered for modelling the damping in the connections, but without success, for, clearly, not being these functions suitable to capture this specific nonlinear behaviour. In fact, due to the friction present in the mounting interfaces, bolted connections may result affected by an hysteretic behaviour, since assembled by using bolted lap-joints. This phenomenon is stressed and put in evidence by the comparison diagram, in which the range from 17 to $21 \mathrm{~Hz}$ appears affected by absence of equivalent solutions, revealing the presence of multiple clearancetype nonlinearities, certainly related to loosening in the bolted attachments. The overall nonlinear damping mechanism behind micro-slips and separation at the interfaces of in-contact surfaces [26,27], cannot, in turn, be modelled by using simple polynomial functions.

The accelerance LeReWi:Y/LeIn:Z of the underlying linear system synthesised by FNSI at model order 44 is compared to the H1 estimator at low level in Fig. 17. The FRF synthesised by the improved FNSI method captures the resonance frequencies well, but it greatly underestimates the amplification factor of modes 4 and 5. To further elucidate the matter, in Fig. 17, we 


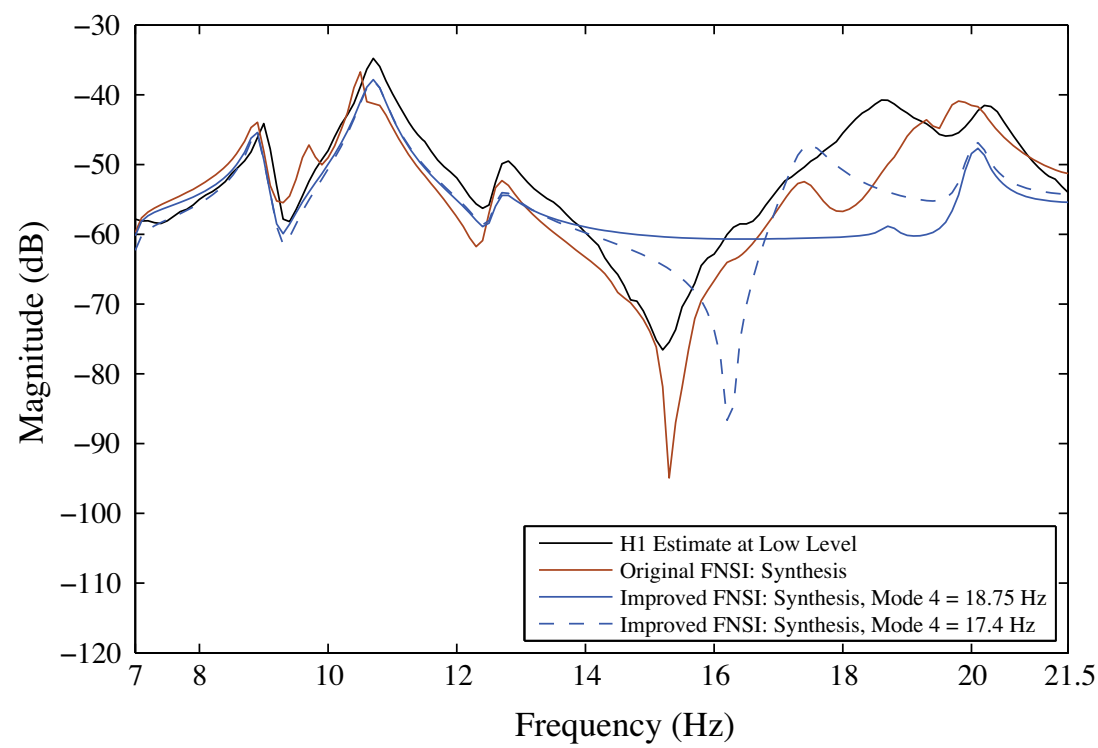

Fig. 17. Experimental model: Accelerance FRF LeReWiY:Z/LeIn:Z computed from high amplitude data (43.9 N RMS). The black curve is obtained through the $\mathrm{H} 1$ estimator (at low excitation level). Syntheses are defined similarly to Fig. 8. (For interpretation of the references to colour in this figure legend, the reader is referred to the web version of this article.)

even offer the comparison with the synthesis computed by the improved FNSI, when the pole 4 , at $18.75 \mathrm{~Hz}$, selected by the comparison approach, is manually replaced with the high level solution, at $17.40 \mathrm{~Hz}$, having the best MAC against the mode 4 identified from low level data (see Table 4). This solution, encircled in blue in Fig. 16, although characterised by a pretty large shift of the natural frequency, is able to provide a better agreement in terms of damping ratio and to make the antiresonance appear between modes 3 and 4 . Nevertheless, we clearly see that even this second synthesis remains far from the H1 low level estimate of the considered FRF, in the high frequency region of the selected range. These results, again, enlighten the influence on nonlinear identification of the adopted basis functions, which seem to be not suitable for capturing or simply compensating some of the nonlinearities that affect the dynamics of this specific full-scale structure.

The presence of spurious poles in the considered frequency range translates into several additional resonances for the FRF synthesised by the original FNSI method. Considering the frequency scheme for the identification of the nonlinear coefficients, the presence of a spurious pole at $10.55 \mathrm{~Hz}$ in Fig. 16 perturbs the nonlinear coefficients identified by the original FNSI method, as shown in Fig. 18a. On the contrary, the influence of this spurious pole is completely removed by the improved FNSI method in Fig. 18b thanks to the model reduction strategy. Moreover, taking advantage of the new extraction scheme, we observe an increased ratio between real and imaginary parts of the resulting nonlinear coefficients.

\section{Conclusions}

The aim of the present paper was to improve the recently-introduced FNSI method by implementing a model reduction strategy for removing spurious poles and by introducing lower and upper frequency residuals, accounting for the influence of out-of-band modes. In particular, spurious pole removal was carried out by operating directly on the identified state-space matrices, basically extending the theoretical concepts of classical modal analysis toward nonlinear mechanical systems. Two different applications, namely the numerical and the experimental model of a full-scale aircraft, were exploited to prove the effectiveness of the proposed methodology. We found out an improved agreement, in both the cases, when comparing the synthesised FRFs and the related modelling errors. In addition, the nonlinear coefficients were identified, accounting for the contribution of different inputs and modes of vibration. The robustness of a new extraction scheme was demonstrated by using the results of the numerical model. Owing to the activation of specific nonlinear phenomena, involving the damping forces, some challenging issues emerged when the proposed methodology was used to process the experimental data. Nevertheless, we found out that the splines on relative displacements, used as basis functions, were able to reliably capture the nonlinear stiffness behaviour of the bolted connections between the wing tips and two external fuel tanks, in the closeness of the wing bending mode. By applying the proposed procedure and taking advantage of the new extraction scheme, we observed an increased ratio between real and imaginary parts of the resulting nonlinear coefficients. Accordingly to the estimated nonlinear restoring forces, we clearly detected the presence of multiple clearance-type nonlinearities, revealing the presence of loosening in the bolted attachments. Thus, the experimental results highlighted that a proper characterisation of the observed nonlinearities, especially in joints like bolted connections, remains of utmost importance before attempting any estimation of nonlinear parameters. 
(a) Original FNSI
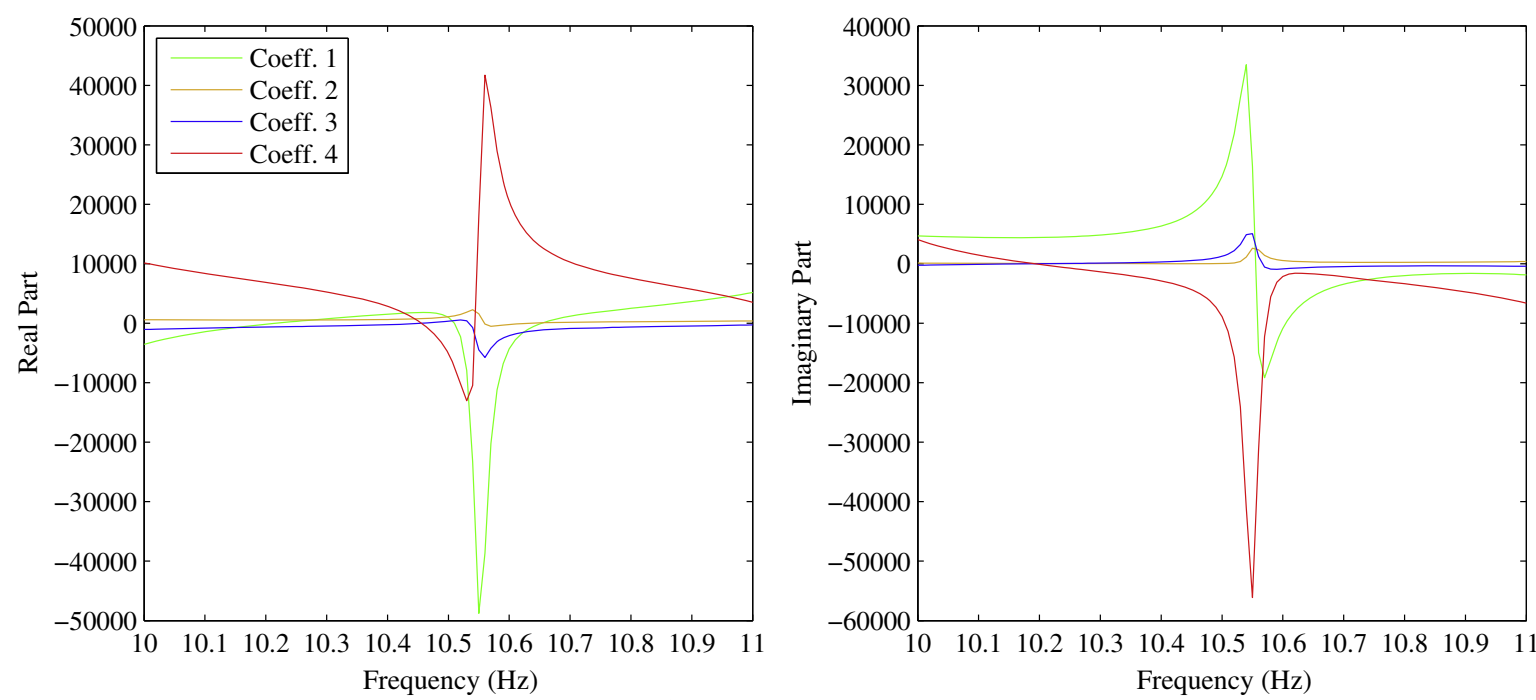

(b) Improved FNSI
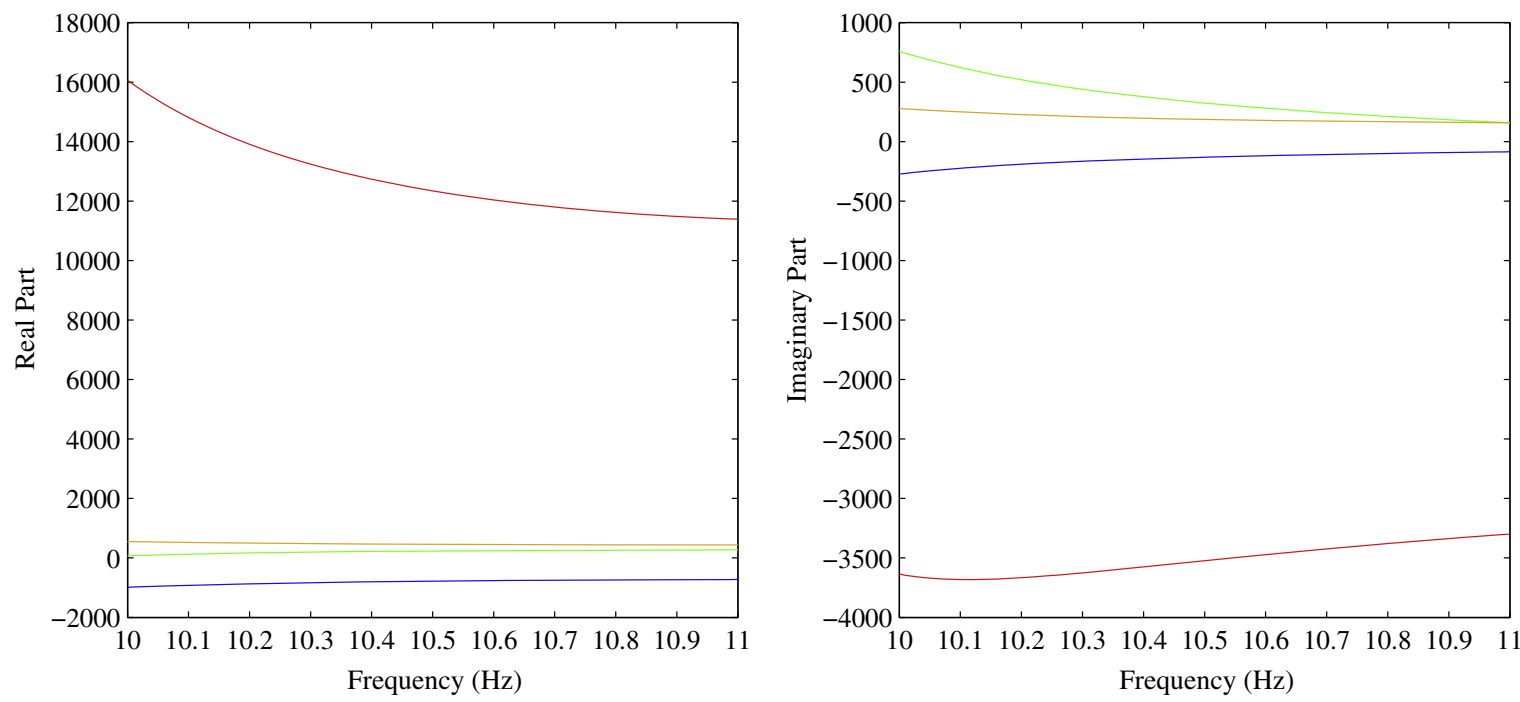

Fig. 18. Nonlinear coefficients related to the output RiReBC:Z and obtained by combining (a) original and (b) improved FNSI with frequency scheme. The estimate is performed in the $10-11 \mathrm{~Hz}$ frequency range, in the closeness of the wing bending mode, at $10.70 \mathrm{~Hz}$ (see Table 4 ). Corresponding modal scheme values, estimated in combination with the improved FNSI, are: Coeff. $1=248.10+\mathrm{i} 217.09$, Coeff. $2=468.94+\mathrm{i} 173.43$, Coeff. $3=-804,02-\mathrm{i} 101.69$, Coeff. $4=12769-\mathrm{i} 3955.0$. (For interpretation of the references to colour in this figure legend, the reader is referred to the web version of this article.)

\section{Acknowledgement}

The author J.P. Noël is a Postdoctoral Researcher of the Fonds de la Recherche Scientifique - FNRS which is gratefully acknowledged. The author G. De Filippis attended a full Doctoral Program at Politecnico di Bari, during which he spent ten months working as visiting researcher at the University of Liège, thanks to a PhD scholarship funded, through the PON01_02380, by the Ministry of Education, University, and Research - MIUR, which is even gratefully acknowledged.

\section{References}

[1] G. Kerschen, K. Worden, A. Vakakis, J. Golinval, Past, present and future of nonlinear system identification in structural dynamics, Mech. Syst. Signal Process. 20 (2006) 505-592.

[2] M. Link, M. Boeswald, S. Laborde, M. Weiland, A. Calvi, Non-linear experimental modal analysis and application to satellite vibration test data, in: Proceedings of the 3rd International Conference on Computational Methods in Structural Dynamics and Earthquake Engineering (COMPDYN), Corfu, Greece, 2011. 
[3] D. Goege, U. Fullekrug, M. Sinapsius, M. Link, Advanced test strategy for identification and characterization of nonlinearities of aerospace structures, AIAA J. 43 (2005) 974-986.

[4] M. Platten, J. Wright, G. Dimitriadis, J. Cooper, Identification of multi-degree of freedom non-linear system using an extended modal space model, Mech. Syst. Signal Process. 23 (2009) 8-29.

[5] M. Weiland, M. Link, Direct parameter estimation of weak nonlinear systems using vibration test data, in: ASME Conference on Mechanical Vibration and Noise, 1995.

[6] M. Weiland, M. Link, A direct parameter estimation method for weak nonlinear systems, in: International Modal Analysis Conference (IMAC) XIV, 1996.

[7] D. Ewins, Modal Testing: Theory, Practice and Application, Research Studies Press, Baldock, United Kingdom, 2000.

[8] B. Peeters, H. Climent, R. de Diego, J. de Alba, J. Ahlquist, J. Carreño, W. Hendricx, A. Rega, G. Garcia, J. Deweer, J. Debille, Modern solutions for Ground Vibration Testing of large aircraft, in: Proceedings of the 26th International Modal Analysis Conference (IMAC), Orlando, FL, USA, 2008.

[9] J.P. Noël, G. Kerschen, Frequency-domain subspace identification for nonlinear mechanical systems, Mech. Syst. Signal Process. 40 (2013) $701-717$.

[10] P. Van Overschee, B. De Moor, Subspace Identification for Linear Systems: Theory, Implementation and Applications, Kluwer Academic Publishers, Dordrecht, The Netherlands, 1996.

[11] T. McKelvey, H.A. cay, L. Ljung, Subspace-based multivariable system identification from frequency response data, IEEE Trans. Autom. Control 41 (7) (1996) 960-979.

[12] E. Reynders, G. De Roeck, Reference-based combined deterministic-stochastic subspace identification for experimental and operational modal analysis, Mech. Syst. Signal Process. 22 (2008) 617-637.

[13] L. Mevel, L. Hermans, H. Van der Auweraer, Application of a subspace-based fault detection method to industrial structures, Mech. Syst. Signal Process. 13 (1999) 823-838.

[14] J.P. Noël, S. Marchesiello, G. Kerschen, Subspace-based identification of a nonlinear spacecraft in the time and frequency domains, Mech. Syst. Signal Process. 43 (2014) 217-236.

[15] J.P. Noël, G. Kerschen, E. Foltête, S. Cogan, Grey-box identification of a nonlinear solar array structure using cubic splines, Int. J. Non-linear Mech. 67 (2014) 106-119.

[16] J.P. Noël, A Frequency-Domain Approach to Subspace Identification of Nonlinear Systems - Application to Aerospace Structures, Ph.D. Thesis, University of Liège, Liège, Belgium, 2014.

[17] S. Marchesiello, A. Fasana, L. Garibaldi, Modal contributions and effects of spurious poles in nonlinear subspace identification, Mech. Syst. Signal Process. 74 (2016) 111-132.

[18] D. Adams, R. Allemang, A frequency domain method for estimating the parameters of a non-linear structural dynamic model through feedback, Mech. Syst. Signal Process. 14 (2000) 637-656.

[19] R. Pintelon, Frequency-domain subspace system identification using non-parametric noise models, Automatica 38 (8) (2002) 1295 -1311.

[20] B. Peeters, System Identification and Damage Detection in Civil Engineering, Ph.D. Thesis, Katholieke Universiteit Leuven, Leuven, Belgium, 2000.

[21] B. Peeters, H. Van der Auweraer, F. Vanhollebeke, P. Guillaume, Operational modal analysis for estimating the dynamic properties of a stadium structure during a football game, Shock Vib. 14 (4) (2007) 283-303.

[22] R. Craig, M. Bampton, Coupling of substructures for dynamic analysis, AIAA J. 6 (1968) 1313-1319.

[23] W. Heylen, S. Lammens, P. Sas, Modal Analysis Theory and Testing, Departement Werktuigkunde, Katholieke Universteit Leuven, Leuven, Belgium, 2007.

[24] L. Soria, B. Peeters, J. Anthonis, H. Van der Auweraer, Operational modal analysis and the performance assessment of vehicle suspension systems, Shock Vib. 19 (5) (2012) 1099-1113.

[25] G. De Filippis, J.P. Noël, G. Kerschen, L. Soria, C. Stephan, Experimental nonlinear identification of an aircraft with bolted connections, in: International Modal Analysis Conference (IMAC) XXXIII, 2015.

[26] H. Jalali, H. Ahmadian, J.E. Mottershead, Identification of nonlinear bolted lap-joint parameters by force-state mapping, Int. J. Solids Struct. 44 (25) (2007) 8087-8105

[27] J.P. Noël, G. Kerschen, Nonlinear system identification in structural dynamics: 10 more years of progress, Mech. Syst. Signal Process. 83 (2017) 2-35. 